\title{
1 An in vivo reporter for tracking lipid droplet dynamics in transparent zebrafish
}

2

3 Dianne Lumaquin ${ }^{1,2, \star}$, Eleanor Johns ${ }^{1,3, \star}$, Joshua Weiss ${ }^{1,2}$, Emily Montal ${ }^{1}$, Olayinka Ooladipupo ${ }^{1}$,

4 Abderhman Abuhashem ${ }^{2,4}$, Richard M. White ${ }^{1}$

5

$6 \quad{ }^{1}$ Cancer Biology and Genetics Program, Memorial Sloan Kettering Cancer Center, New York, 7 NY, USA

$8{ }^{2}$ Weill Cornell/Rockefeller/Sloan Kettering Tri-Institutional MD-PhD Program, Memorial Sloan

9 Kettering Cancer Center, New York, NY, USA

$10{ }^{3}$ Gerstner Sloan Kettering Graduate School of Biomedical Sciences, Memorial Sloan Kettering

11 Cancer Center, New York, NY, USA

$12{ }^{4}$ Developmental Biology Program, Memorial Sloan Kettering Cancer Center, New York, NY,

13 USA

14 * These authors contributed equally to this work 


\section{Abstract}

16 Lipid droplets are lipid storage organelles found in nearly all cell types from adipocytes to cancer

17 cells. Although increasingly implicated in disease, current methods to study lipid droplets require

18 fixation or static imaging which limits investigation of their rapid in vivo dynamics. To address this,

19 we created a lipid droplet transgenic reporter in whole animals and cell culture by fusing

20 tdTOMATO to Perilipin-2 (PLIN2), a lipid droplet structural protein. Expression of this transgene

21 in transparent casper zebrafish enabled in vivo imaging of adipose depots responsive to nutrient

22 deprivation and high-fat diet. Using this system, we tested novel regulators of lipolysis, revealing

23 an unexpected role for nitric oxide in modulating adipocyte lipid droplets. Similarly, we expressed

24 the PLIN2-tdTOMATO transgene in melanoma cells and found that the nitric oxide pathway also

25 regulated lipid droplets in cancer. This model offers a tractable imaging platform to study lipid

26 droplets across cell types and disease contexts. 


\section{Introduction}

28 Lipid droplets are cellular organelles which act as storage sites for neutral lipids and are key

29 regulators of cellular metabolism (Farese \& Walther, 2009). Lipid droplets are present in most

30 cell types and are characterized by a monophospholipid membrane surrounding a hydrophobic

31 lipid core (Olzmann \& Carvalho, 2019). Cells maintain energetic homeostasis and membrane

32 formation through the regulated incorporation and release of fatty acids and lipid species from

33 the lipid droplet core (Jarc \& Petan, 2019; Olzmann \& Carvalho, 2019). Importantly, lipid

34 droplets can assume various functions during cellular stress through the sequestration of

35 potentially toxic lipids and misfolded proteins, maintenance of energy and redox homeostasis,

36 regulation of fatty acid transfer to the mitochondria for $\beta$-oxidation, and the maintenance of ER

37 membrane homeostasis (Olzmann \& Carvalho, 2019; Petan et al., 2018). Moreover, recent work

38 demonstrated that lipid droplets actively participate in the innate immune response (Bosch et al.,

39 2020), and conversely, can be hijacked by infectious agents like hepatitis C virus to facilitate

40 viral replication (Barba et al., 1997; Miyanari et al., 2007; Vieyres et al., 2020). The role of lipid

41 droplets in metabolic homeostasis and cellular stress is critical across multiple cell types and

42 has also been increasingly implicated in cancer (Petan et al., 2018). For example, lipid droplets

43 can act as a storage pool in cancer cells after they take up lipids from extracellular sources,

44 including adipocytes (Kuniyoshi et al., 2019; Nieman et al., 2011; Zhang et al., 2018).

46 While lipid droplets are ubiquitous across most cell types, they are essential to the function of

47 adipocytes in regulating organismal energy homeostasis (Jarc \& Petan, 2019). White adipocytes

48 contain a large unilocular lipid droplet that is tightly regulated to mobilize fatty acids from the

49 lipid droplet core (Heid et al., 2014; Zechner et al., 2017). Activation of lipolysis releases free

50 fatty acids from the adipocyte lipid droplet which can be used by surrounding, non-adipose cell

51 types to fuel energy production (Schoiswohl et al., 2010; Zimmermann et al., 2004).

52 Dysregulation of lipid droplet function has been linked to a variety of pathophysiologies namely 
53 obesity (Olzmann \& Carvalho, 2019). In addition, mutations in lipases required for lipolysis can

54 lead to increased fat deposition and systemic metabolic abnormalities (Ahmadian et al., 2011;

55 Haemmerle et al., 2006; Schoiswohl et al., 2010) in mouse models as well as the development

56 of neutral lipid storage disease in humans (Fischer et al., 2007).

58 In vivo imaging of lipid droplets, either in adipocytes or in other cell types, is currently highly

59 limited. Understanding these dynamics in vivo, rather than in fixed tissues, is important since the

60 size of the lipid droplet can change very rapidly in response to fluctuating metabolic needs

61 (Bosch et al., 2020; Fam et al., 2018). Much of adipose tissue imaging utilizes tissue fixation

62 and sectioning, which can fail to preserve key aspects of the tissue structure (Berry et al., 2014;

63 Xue et al., 2010). Whole mount imaging approaches in mice can be combined with adipocyte

64 specific promoters, however, these methods still require tissue dissection and can be limited by

65 tissue thickness (Berry \& Rodeheffer, 2013; Chi et al., 2018).

67 Zebrafish offer a tractable model to address these limitations given the ease of high-throughput

68 imaging of live animals. This is especially true with the availability of relatively transparent

69 strains such as casper, which allow for detailed in vivo imaging without the need for fixation of

70 the animal (White et al., 2008). Although less well studied than other vertebrates, zebrafish

71 adipose tissue is highly similar to mammalian white adipose tissue and detailed work has

72 classified the timing, dynamics, and location of zebrafish adipose tissue development (Minchin

73 \& Rawls, 2017). However, until now, the study of zebrafish adipose tissue has been limited to

74 the use of lipophilic fluorescent dyes, which are restricted in their ability to read out dynamic

75 changes over long periods of time (Fam et al., 2018).

77 Here, we report the development of an in vivo lipid droplet reporter using a plin2-tdtomato

78 transgene in the casper strain. To date, transgenic lipid droplet reporters have been restricted to 
79 invertebrate model organisms such as C. Elegans and Drosophila (Kühnlein, 2011; Liu et al.,

80 2014). We demonstrate that the reporter faithfully marks the lipid droplet which enables robust

81 in vivo imaging. We show that this reporter can be applied to visualize adipocytes and to

82 monitor adipose tissue remodeling in response to dietary and pharmacologic perturbations.

83 Furthermore, we report the discovery of novel pharmacologic regulators of adipocyte lipolysis

84 such as nitric oxide and demonstrate that several of these compounds can modulate adipose

85 tissue area in our in vivo system. To facilitate the study of lipid droplets in novel contexts outside

86 of adipocytes, we also generated a zebrafish melanoma cell line (ZMEL) (Heilmann et al., 2015)

87 expressing plin2-tdtomato (ZMEL-LD). We confirm that this cell line can be used to monitor

88 changes in lipid droplet production in response to both known and novel regulators of lipolysis.

89 We anticipate that these models will be highly valuable as a high-throughput imaging platform to

90 investigate lipid droplets in both adipose tissue biology as well as disease contexts such as

91 cancer. 


\section{Results}

\section{An in vivo lipid droplet reporter using a PLIN2-tdTOMATO fusion transgene}

94 To create a specific, fluorescent reporter for lipid droplets in zebrafish, we fused tdtomato to the

95 3' end of the plin2 cDNA. We chose plin2 because it is a well-known lipid droplet associated

96 protein that is ubiquitously expressed on lipid droplets across cell types (Olzmann \& Carvalho,

97 2019). We generated stable transgenic zebrafish expressing ubb:plin2-tdtomato and sought to

98 validate whether the construct faithfully marks lipid droplets (Figure 1A). White adipocytes are

99 fat cells known for their large unilocular lipid droplet (T. Fujimoto \& Parton, 2011; Heid et al.,

100 2014) so we expected expression of the PLIN2-tdTOMATO fusion protein on the surface of the

101 adipocyte lipid droplet (Figure 1A). Since the adipocyte lipid droplet occupies the majority of

102 space in the cell (M. Fujimoto et al., 2020), existing methods to visualize zebrafish adipocytes

103 rely on lipophilic dyes and lipid analogs which incorporate in the lipid droplet (Zhang et al.,

104 2018). Thus in addition to labeling individual lipid droplets, we reasoned that the PLIN2-

105 tdTOMATO fusion protein can also function as a reporter for adipocytes since these cells would

106 have the largest and unilocular lipid droplets.

In adult zebrafish, subcutaneous adipocytes are known to reside proximally to the tail fin (Minchin \& Rawls, 2017). When we imaged six month old adult tg(ubb:plin2-tdtomato) zebrafish, we detected PLIN2-tdTOMATO expression in the zebrafish tail fin adipocytes which colocalizes with BODIPY staining (Figure 1B, C). Lipophilic dyes such as BODIPY stain the lipid-rich core of

112 the lipid droplet while lipid droplet resident proteins, such as PLIN2, localize to the lipid droplet

113 membrane (Zhang et al., 2018). As expected, higher magnification images of tail adipocytes

114 revealed that PLIN2-tdTOMATO expression was on the outside of the lipid droplet, whereas the

115 BODIPY staining was on the interior of each droplet in the adipocyte (Figure 1D). Similarly,

116 immunohistochemistry on the $\operatorname{tg}(u b b: p l i n 2-t d t o m a t o)$ zebrafish tail fin showed that adipocytes

117 express tdTOMATO (Figure 1E). Taken together, this data demonstrates that the PLIN2- 
tdTOMATO fusion protein functions as a fluorescent lipid droplet reporter which can be applied to visualize adipocytes in vivo.

\section{The $\operatorname{tg}$ (ubb:plin2-tdtomato) is an in vivo reporter for visceral adipocytes}

122 Visceral adipose tissue, otherwise known as abdominal fat, plays an important role in

123 metabolism and participates in pathological processes of obesity, aging and metabolic

124 syndromes (Tchernof \& Després, 2013). Because PLIN2-tdTOMATO labeled subcutaneous

125 adipocytes in the adult zebrafish tail fin, we wondered whether we could use the $\operatorname{tg}(u b b$ :plin2-

126 tdtomato) zebrafish to visualize other adipose depots in vivo such as visceral adipocytes. In

127 juvenile zebrafish at 21 days post-fertilization (dpf), visceral adipose tissue is composed of

128 abdominal and pancreatic visceral adipocytes predominantly located on the right flank near the

129 swim bladder (Figure 2A) (Minchin \& Rawls, 2017). To determine whether tg(ubb:plin2-

130 tdtomato) visceral adipocytes express PLIN2-tdTOMATO, we imaged around the swim bladder

131 of juvenile zebrafish where we expect development of abdominal visceral adipocytes (Figure

132 2B). Visceral adipocytes visualized in brightfield co-stain for PLIN2-tdtomato and BODIPY, as

133 we observed for subcutaneous adipocytes (Figure 2C). Immunohistochemistry of the juvenile

$134 \operatorname{tg}(u b b: p l i n 2-t d t o m a t o)$ confirmed that the abdominal and visceral adipocytes express

135 tdTOMATO (Figure 2D). Combined with the ability for high-throughput in vivo imaging in

136 zebrafish, we sought to use $\operatorname{tg}(u b b$ :plin2-tdtomato) as a model to study lipid droplet dynamics in

137 visceral adipocytes. One challenge we encountered was the auto-fluorescence from the

138 zebrafish intestinal loops and gallbladder present in the tdTOMATO and GFP channels (Figure

$1392 \mathrm{E}$ ). To remove background fluorescence, we developed an image analysis pipeline in MATLAB

140 to segment the visceral adipocytes in the juvenile $\operatorname{tg}(u b b$ :plin2-tdtomato) (Figure 2E). Thus,

$141 \operatorname{tg}$ (ubb:plin2-tdtomato) can be used as an in vivo model to visualize adipocytes with the benefits

142 of avoiding staining steps and allowing for high-throughput image analysis in zebrafish. 


\section{Diet and pharmacologically induced reduction in visceral adipose tissue area}

144 After confirming that we could image visceral adipose tissue in $\operatorname{tg}$ (ubb:plin2-tdtomato), we

145 wanted to test whether this could be a tractable platform to image adipose tissue remodeling.

146 We first verified whether we could use tg(ubb:plin2-tdtomato) to track reduction in visceral

147 adiposity. Fasting is a well-known mechanism for reducing adiposity, since it will induce lipolysis

148 and lead to a reduction in the size of the adipocyte lipid droplet (Henne et al., 2018; Longo \&

149 Mattson, 2014; Rambold et al., 2015; Tang et al., 2017). To test this, juvenile zebrafish were

150 given control feed or fasted for 2.5 days then imaged to measure standard length and adipose

151 tissue area (Figure 3A). As expected, we observed a reduction in the segmented adipocyte area

152 in the fasted zebrafish (Figure 3B). Using our image analysis pipeline, we measured a

153 significant reduction in adipose tissue area with an average of $0.39 \pm 0.03 \mathrm{~mm}^{2}$ for fed fish and

$1540.21 \pm 0.03 \mathrm{~mm}^{2}$ for fasted fish (Figure $3 \mathrm{C}$ ). The control fed fish had a longer average standard

155 length compared to the fasted fish $(9.76 \pm 0.18 \mathrm{~mm}$ vs $8.73 \pm 0.17 \mathrm{~mm})$ which we attribute to

156 food restriction disrupting zebrafish development during this developmental window (Figure 3D).

157 We saw a similar reduction in fasted fish when normalizing adipose tissue area to standard

158 length, similar to a Body Mass Index (BMI) in mammals (control feed $=0.040 \pm 0.003$ area/SL

159 and fasted $=0.024 \pm 0.003$ area/SL) (Figure 3E).

161 In addition to fasting as a dietary perturbation, we also pharmacologically reduced adipose

162 tissue. To achieve this, we used Forskolin, a drug which is known to induce lipolysis through

163 cAMP signaling (Litosch et al., 1982). We treated juvenile zebrafish for 24 hours with either

164 DMSO or $5 \mu \mathrm{M}$ Forskolin and imaged the adipocytes (Figure 3F). We detected a reduction in

165 both the adipose tissue area and normalized area to standard length in the Forskolin treated

166 fish, but no differences in standard length (Figure 3G, H, I). Altogether, this data suggests that

167 our PLIN2-tdTOMATO reporter faithfully reads out changes in the size of adipose tissue due to

168 its capacity to sensitively detect lipolysis of the large lipid droplet in this tissue. 


\section{High-fat diet leads to specific enlargement of visceral adipose tissue}

171 Having shown that we could use tg(ubb:plin2-tdtomato) to image and measure reduction in

172 adipose tissue, we tested whether we can use our model to detect an increase in adiposity.

173 Zebrafish have been used as a model for diet-induced obesity and share pathophysiological

174 perturbations seen in mammals, but few studies have focused on architectural changes of

175 visceral adipose tissue (Chu et al., 2012; Landgraf et al., 2017; Oka et al., 2010). We sought to

176 determine if we could detect increases in visceral adiposity from a high fat diet (HFD). We fed

177 juvenile zebrafish with either control feed ( $12 \%$ crude fat) or HFD ( $23 \%$ crude fat) for 7 days and

178 subsequently imaged the adipose tissue (Figure 4A, B). Remarkably after a week of HFD

179 feeding, we observed that HFD fed fish developed notably increased visceral adiposity

180 compared to the fish fed with control feed (Figure 4C). Quantification of the adipose tissue

181 revealed that HFD led to an increase in adipose tissue area (control feed $0.42 \pm 0.03 \mathrm{~mm}^{2}$ and

$182 \mathrm{HFD}=0.62 \pm 0.03 \mathrm{~mm}^{2}$ ) and normalized area to standard length (control feed $=0.040 \pm 0.002$

183 area/SL and HFD $=0.061 \pm 0.002$ area/SL) (Figure 4D, F). Interestingly, we did not detect

184 differences in the standard length of the fish (control feed $=10.09 \pm 0.11 \mathrm{~mm}$ and HFD $=10.13$

$185 \pm 0.11 \mathrm{~mm}$ ), suggesting that this formulation of HFD leads to specific enlargement of visceral

186 adipose tissue (Figure 4E). Our results demonstrate that $\operatorname{tg}$ (ubb:plin2-tdtomato) is an effective

187 and unique tool to visualize visceral adipose tissue remodeling induced by HFD which can be

188 widely applied to study obesity.

A screen to discover novel compounds that modulate lipolysis and lipid droplets in vivo

191 To meet fluctuating nutritional needs of the cell, lipid droplets are remodeled through lipolysis to

192 regulate lipid mobilization and metabolic homeostasis (Krahmer et al., 2013; Olzmann \&

193 Carvalho, 2019; Paar et al., 2012). As a major lipid depot for the body, white adipose tissue is

194 critical to lipid availability and cycles through lipolytic flux in response to energy demands 
195 (Duncan et al., 2007). In disease contexts such as cancer, adipocytes undergoing lipolysis act

196 as a lipid source for neighboring cancer cells (Lengyel et al., 2018). Adipocyte-derived lipids

197 have been directly shown to promote cancer progression in ovarian (Nieman et al., 2011),

198 breast (Balaban et al., 2017), and melanoma cancer cells (Zhang et al., 2018). Due to growing

199 evidence of adipocyte and cancer cell cross-talk as a metabolic adaptation for tumor

200 progression, there is significant interest in disrupting lipid transfer between adipocytes and

201 cancer cells.

202

203 Leveraging our model to visualize lipid droplets in adipocytes, we became interested in

204 identifying novel compounds that remodel adipocyte lipid droplets through lipolysis. In

205 mammalian systems, the most commonly used cell line to study lipolysis are 3T3-L1 cells, which

206 can be differentiated in vitro to resemble adipocytes (Zebisch et al., 2012). We first used the

207 3T3-L1 system to rapidly identify lipolysis inhibitors at high-throughput, and then test those hits

208 using our zebrafish lipid droplet reporter. We reasoned that compounds which inhibit lipolysis in

209 vitro would cause an increase in the size of the lipid droplets in vivo. To achieve this, we

210 differentiated mouse 3T3-L1 fibroblast cells into adipocytes and conducted a chemical screen

211 for compounds that inhibit lipolysis (Figure 5A), measured by quantifying glycerol in the media, a

212 gold standard readout of lipolysis in this system (Hellmér et al., 1989). As a positive control, we

213 used Atglistatin, an inhibitor of adipose triglyceride lipase (ATGL) which is known to be the rate

214 limiting step of lipolysis and has been shown to inhibit lipolysis in cell lines and mouse models

215 (Mayer et al., 2013; Schweiger et al., 2017). We confirmed that Atglistatin potently inhibits

216 lipolysis in 3T3-L1 adipocytes (Figure 5B). We then screened through a library of 1,280

217 compounds of diverse chemical structures to find novel inhibitors of lipolysis. Overall, we found

21829 out of 1,280 compounds which led to at least a $40 \%$ reduction in lipolysis as measured by

219 glycerol release into the media. Looking more closely at the top 10 hits from this screen, we

220 noted that 2 of the top 10 top hits (Auranofin and JS-K), both modulated nitric oxide (Figure 5A). 
221 Nitric oxide can be used for post-translational modification of proteins via S-nitrosylation

222 (Stamler et al., 2001). Previous work has shown that increased nitric oxide has a suppressive

223 role on lipolysis, and Auranofin, a thioredoxin reductase inhibitor that promotes S-nitrosylation,

224 can inhibit lipolysis in 3T3-L1 cells (Yamada et al., 2015). Similarly, JS-K is a nitric oxide donor

225 purported to promote S-nitrosylation, but it has not been shown to play a role in lipolysis (Nath

226 et al., 2010; Shami et al., 2003). Given that both of these top hits were in the same pathway, we

227 chose these for in vivo validation. We asked whether these drugs could modulate lipid droplet

228 size and lead to increased adiposity in the zebrafish. We treated juvenile zebrafish for 24 hours

229 with DMSO, Atglistatin, Auranofin, or JS-K and imaged the adipose tissue. We found that

230 Atglistatin and JS-K significantly increase adipose tissue area and normalized area to standard

231 length (Figure 5C, E). These effects were specific to the adipose tissue as standard length was

232 not affected (Figure 5D). These data indicate that modulators of nitric oxide can inhibit lipolysis

233 in cell lines, which then leads to an increase in adipose tissue area in vivo in the zebrafish.

234 Moreover, this approach demonstrates the power of this system to dissect the relationship

235 between novel modulators of lipolysis (i.e. nitric oxide) and adiposity in vivo.

237 Lipolysis modulators also inhibit lipid droplet loss in melanoma cells

238 Upon uptake of adipocyte-derived lipids, cancer cells can store excess lipids in lipid droplets

239 (Lengyel et al., 2018). Accumulation of lipid droplets in melanoma cells has been associated

240 with increased metastatic potential and worse clinical outcomes (M. Fujimoto et al., 2020; Zhang

241 et al., 2018). The mechanisms regulating subsequent lipolysis from the lipid droplets in cancer

242 cells are not well understood, but we reasoned that some of the same mechanisms (i.e. ATGL,

243 nitric oxide) used in adipocytes might also be used in cancer cells. To test this, we created a

244 stable zebrafish melanoma cell line (ZMEL) that expressed the ubb:plin2-tdtomato construct

245 (Heilmann et al., 2015) to generate the ZMEL-LD (lipid droplet) reporter cell line (Figure 6A).

246 Because melanoma cells at baseline only have few small lipid droplets, we induced their 
247 formation via extrinsic addition of oleic acid, a key fatty acid that can be transferred from the

248 adipocyte to the melanoma cell (Zhang et al., 2018). We found that after a pulse of oleic acid for

24924 hours, we could easily detect PLIN2-tdTOMATO expression surrounding lipid droplets

250 marked by the lipid droplet dye MDH (Figure 6B). A 3D reconstruction demonstrated that PLIN2-

251 tdTOMATO was strictly expressed on the outline of the lipid droplet core, consistent with

252 endogenous PLIN2 protein expression patterns (Olzmann \& Carvalho, 2019) (Movie 1) and

253 similar to what we saw in the adipocytes (Figure 1).

255 To validate whether lipolysis inhibiting compounds could modulate lipid droplets in the ZMEL-LD

256 cells, we utilized flow cytometry to measure PLIN2-tdTOMATO expression. We treated ZMEL-

257 LD cells for 72 hours with either BSA or oleic acid as controls for low or high lipid droplet cell

258 populations (Figure 6C), which confirmed the ability of the transgene to read out lipid droplets in

259 this assay. We then tested the effects of the lipolysis inhibitors described above. We pulsed the

260 ZMEL-LD cells with oleic acid for 24 hours (to induce lipid droplets) and then measured the

261 subsequent decay in signal over the ensuing 48 hours, which is expected to decrease due to

262 gradual lipolysis of the lipid droplets. Compared to cells with oleic acid pulse and DMSO (58.5 \pm

$2631.4 \%$ LD+ cells $)$, cells given JS-K $(58.3 \pm 1.2 \%$ LD+ cells $)$ did not differ in the percent of lipid

264 droplet positive cells (Figure 6D, E). In contrast, cells treated with Atglistatin $(66.7 \pm 1.4 \%$ LD+

265 cells) and Auranofin (69.1 $\pm 2.1 \%$ LD+ cells) demonstrated significantly higher lipid droplet

266 positive cells (Figure 6D, E). These data indicate that similar to adipocytes, ATGL is a key

267 regulatory step in lipolysis in the melanoma cells. Moreover, we find that nitric oxide, which was

268 identified in our adipocyte screen, is similarly a modulator of lipolysis in the melanoma context

269 and can be utilized for future studies to target adipocyte-melanoma cell cross-talk. We do not

270 yet understand why different nitric oxide donors are more or less potent in adipocytes (where

271 JS-K is a better inhibitor in vivo) versus melanoma cells (where Auranofin is a better inhibitor),

272 but this could reflect differences in pharmacokinetics between the two cell types. 


\section{Discussion}

275 Lipid droplets are cytosolic storage organelles for cellular lipids which are dynamically regulated

276 in response to metabolic and oxidative perturbations (Jarc \& Petan, 2019). For instance, under

277 hypoxic conditions, lipid droplets are crucial for protecting cells against reactive oxygen species

278 and lipid peroxidation (Bailey et al., 2015; Bensaad et al., 2014). Lipid droplets can also buffer

279 ER stress by sequestering excess lipids and proteins in the lipid droplet core (Chitraju et al.,

280 2017; Velázquez et al., 2016; Vevea et al., 2015) while fluctuations in nutrient availability have

281 been shown to lead to changes in lipid droplet biogenesis (Cabodevilla et al., 2013; Nguyen et

282 al., 2017). The regulatory mechanisms driving these processes remain incompletely

283 understood. Furthermore, lipid droplets are highly heterogeneous and the pathways which

284 regulate lipid droplet dynamics in specific cell types warrant investigation.

286 To address such questions, we developed the first lipid droplet reporter in a vertebrate model

287 organism. We show that our plin2-tdtomato reporter faithfully marks the lipid droplet in vivo. The

288 combination of this reporter with the in vivo system of the casper zebrafish enables flexible and

289 robust imaging approaches to examine lipid droplet regulation and function. In particular, the

290 ease of chemical and genetic manipulation of the zebrafish combined with high-throughput

291 imaging approaches enables interrogation of relevant pathways in a cell type specific manner.

292 Furthermore, the capacity for intravital imaging creates the opportunity to conduct longitudinal

293 analysis of lipid droplet dynamics across developmental time and in disease contexts between

294 single animals.

296 Here, we demonstrate the capabilities of the $\operatorname{tg}(u b b$ :plin2-tdtomato) line by taking advantage of

297 the fact that white adipocytes, which are primarily composed of a large unilocular lipid droplet

298 (T. Fujimoto \& Parton, 2011), are readily labeled by PLIN2-tdTOMATO expression. This labeling 
enables the study of individual adipocytes and adipose tissue in adult and juvenile zebrafish.

We utilized this system to develop a robust imaging platform to specifically study the regulation

301 of adipose tissue using both diet and pharmacologic perturbation. We focused on visceral

302 adipose tissue due to its role as an endocrine organ and central regulator of organismal

303 metabolism. Importantly, visceral adipose tissue accumulation, such as in obesity, influences

304 the development of disorders including insulin resistance, cardiovascular disease, and

305 hypertension (Fox et al., 2007; Le Jemtel et al., 2018; Verboven et al., 2018). We use our image

306 analysis pipeline to demonstrate that our model is sensitive to diet induced changes in visceral

307 adiposity. We also show that established chemical regulators of adipocyte lipolysis, Forskolin

308 and Atglistatin, can produce quantitative changes in visceral adipose tissue. Collectively, these

309 data illustrate the potential of our model to yield novel insights into the regulation of visceral

310 adipose tissue, including in the context of obesity.

312 Although adipocytes comprise a major portion of adipose tissue, adipose tissue also consists of

313 the stromal vascular fraction composed of fibroblasts, endothelial, and immune cells (Rosen \&

314 Spiegelman, 2014). Remodeling of adipose tissue architecture through changes in

315 vascularization or recruitment of immune cells during tissue inflammation is associated with

316 metabolic diseases including obesity and insulin resistance (Rosen \& Spiegelman, 2014). Given

317 the complexity of adipose tissue organization, understanding the native tissue architecture in

318 relevant contexts is essential. We anticipate that our reporter can be easily crossed with other

319 zebrafish transgenic reporters of interest to visualize heterotypic cell-cell interactions within

320 adipose tissue.

322 While our studies show that this tool can be readily used to increase our understanding of 323 adipocyte biology, it can also be utilized to study lipid droplets in other contexts as well. 
324 Lipid droplets are ubiquitous across almost all cell types. Therefore, this model could be applied

325 to study the regulation of lipid droplets in the development and function of other adipose depots

326 and additional cell types, such as muscle and hepatocytes (Bosma, 2016; Wang et al., 2013). In

327 the disease context, we focused on the role of lipid droplets in cancer, since they have been

328 implicated in various tumor types (Petan et al., 2018) where tumor cells can take up lipids from

329 adipocytes and then package them into lipid droplets in the cancer cell (Balaban et al., 2017;

330 Lengyel et al., 2018; Nieman et al., 2011; Zhang et al., 2018). This transfer of lipids has been

331 linked to disease progression, making the regulation of lipid release from the lipid droplet

332 through subsequent lipolysis in the tumor cell of particular interest. By expressing the plin2-

333 tdtomato transgene in the ZMEL melanoma cells, we find that key regulators of lipolysis, such

334 as ATGL and nitric oxide are mechanisms conserved with normal adipocytes. Interestingly, our

335 results suggest that while the nitric oxide pathway can alter both adipose tissue area and lipid

336 droplet content in melanoma cells, there may be differences between the phenotypes induced

337 by nitric oxide production compared to more downstream effects such as S-nitrosylation, which

338 are cell type specific. Collectively, this underscores the complexity of lipid droplet regulation and

339 emphasizes the importance of studying these processes in both cell types. We believe that our

340 model will serve as a powerful tool to study cell type specific regulation of lipid droplet

341 biogenesis and function while preserving the endogenous structural and metabolic environment

342 of an in vivo system. 


\section{Acknowledgements}

345 We thank members at the Memorial Sloan Kettering Cancer Center Aquatics Core, Molecular

346 Cytology Core, and Flow Cytometry Core for their contributions to this work. We thank Dr.

347 Mohita Tagore and Dr. Ting-Hsiang (Richard) Huang for comments on the project and

348 manuscript.

\section{Competing Interests}

351 D.L., E.J., J.W., E.M., O.O., and A.A. do not have competing interests to declare. R.M.W is a

352 paid consultant to N-of-One Therapeutics, a subsidiary of Qiagen. R.M.W is on the scientific

353 advisory board of Consano, but receives no income for this. R.M.W receives royalty payments

354 for the use of the casper zebrafish line from Carolina Biologicals. 
A

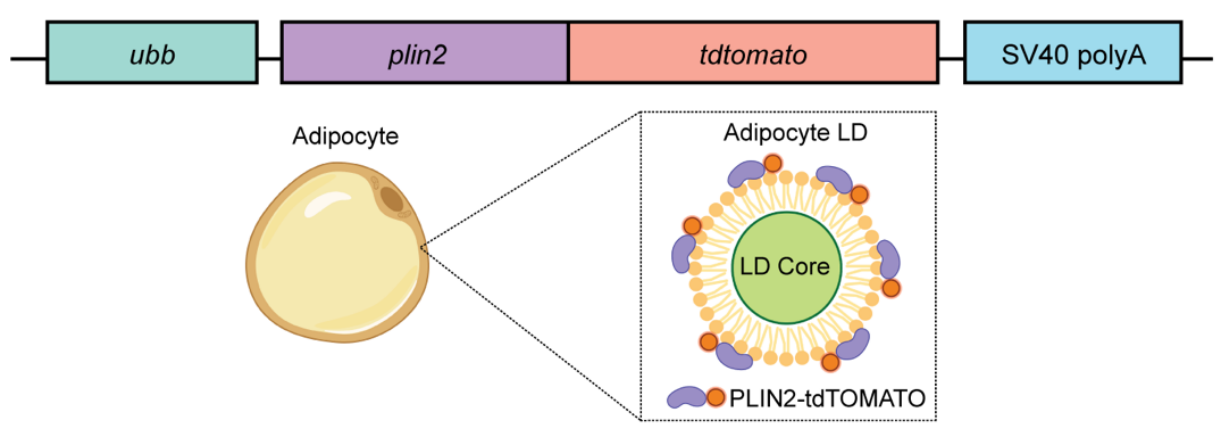

B

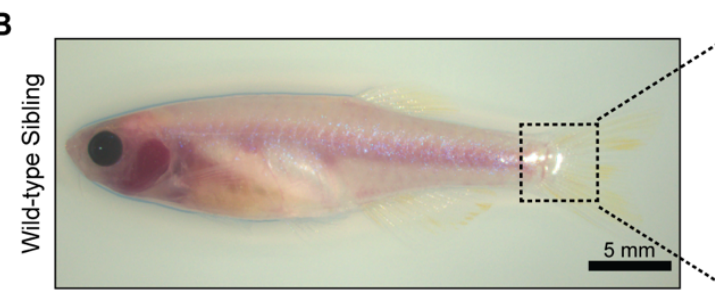

C

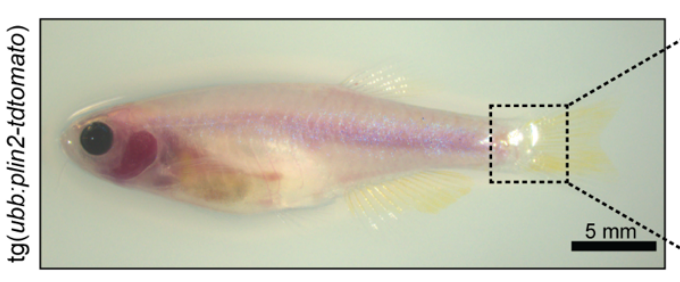

D
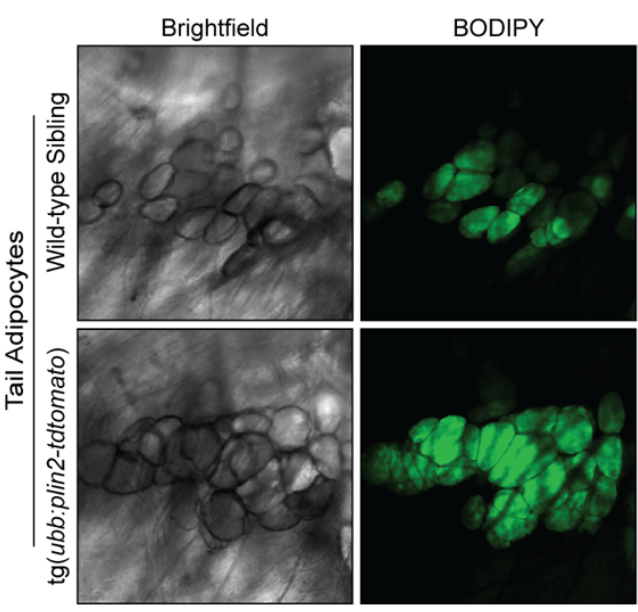
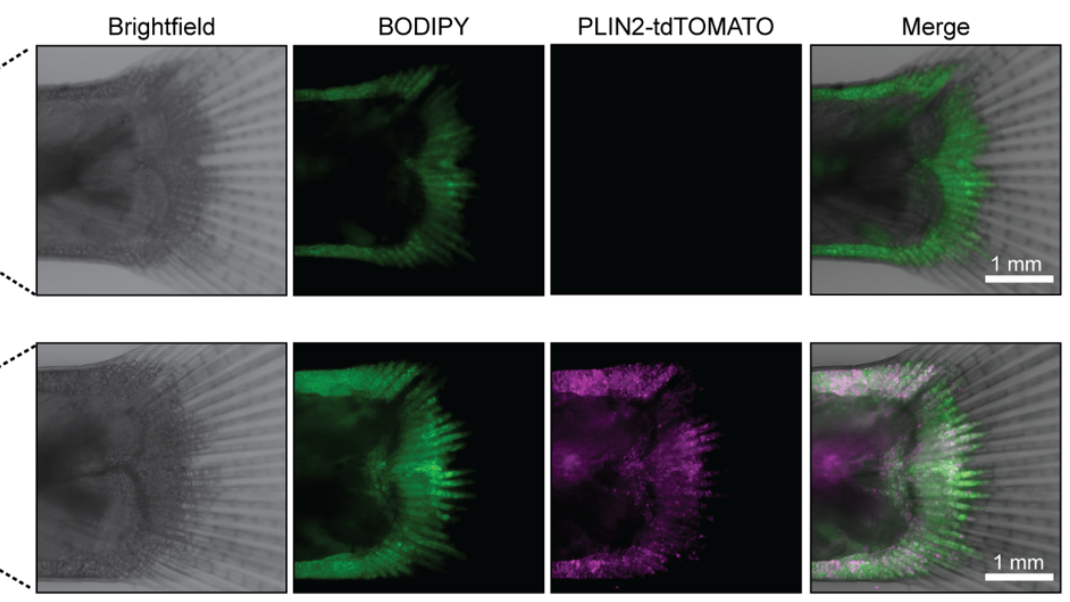

E
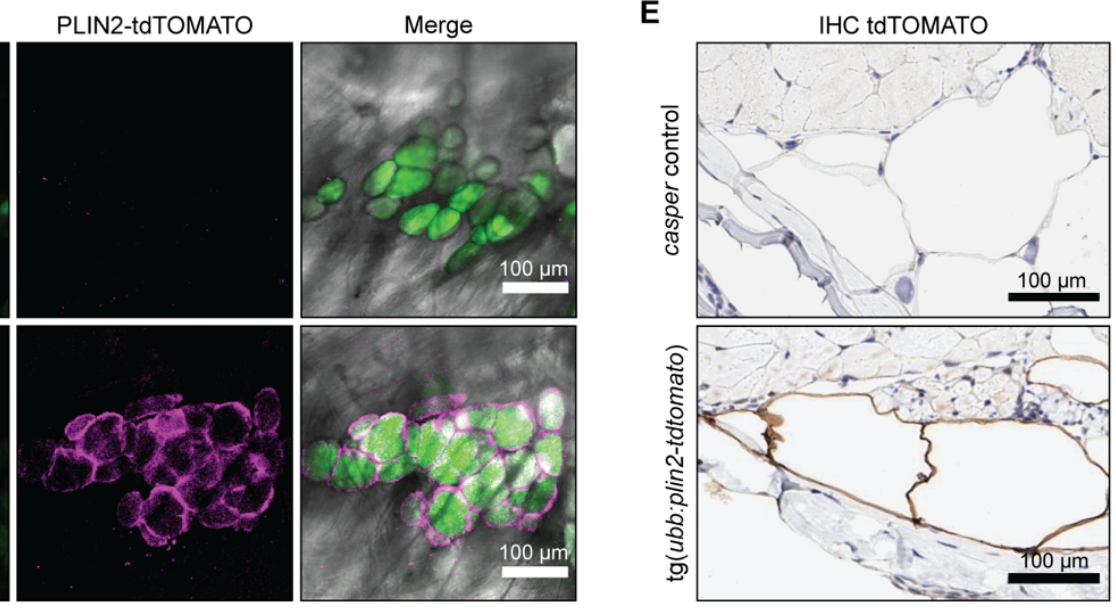

355

\section{Figure 1: An in vivo lipid droplet reporter using a PLIN2-tdTOMATO fusion transgene}

(A) Schematic of ubb:plin2-tdtomato construct injected into zebrafish and adipocyte lipid droplet labeled with PLIN2-tdTOMATO fusion protein. Widefield microscope images of adult (B) wild-type sibling and (C) $\operatorname{tg}$ (ubb:plin2-tdtomato) zebrafish. Box shows zoomed images of the fish tail with panels for brightfield, BODIPY, PLIN2-tdTOMATO, and merged. (D) Confocal images of fish tail adipocytes of adult wild-type sibling and $\operatorname{tg}$ (ubb:plin2-tdtomato) zebrafish. Panels show brightfield, BODIPY, PLIN2-tdTOMATO, and merge. (E) Adult casper and tg(ubb:plin2-tdtomato) zebrafish tails were fixed and immunohistochemistry conducted for tdTOMATO expression of tail adipocytes. 
A

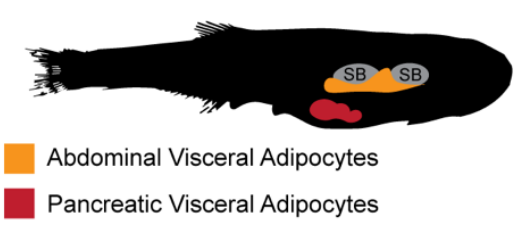

B

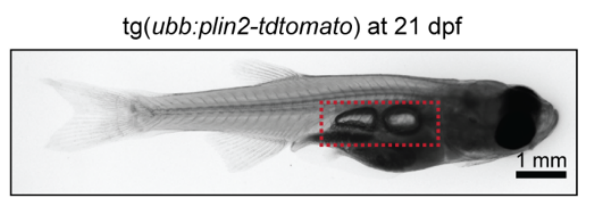

C
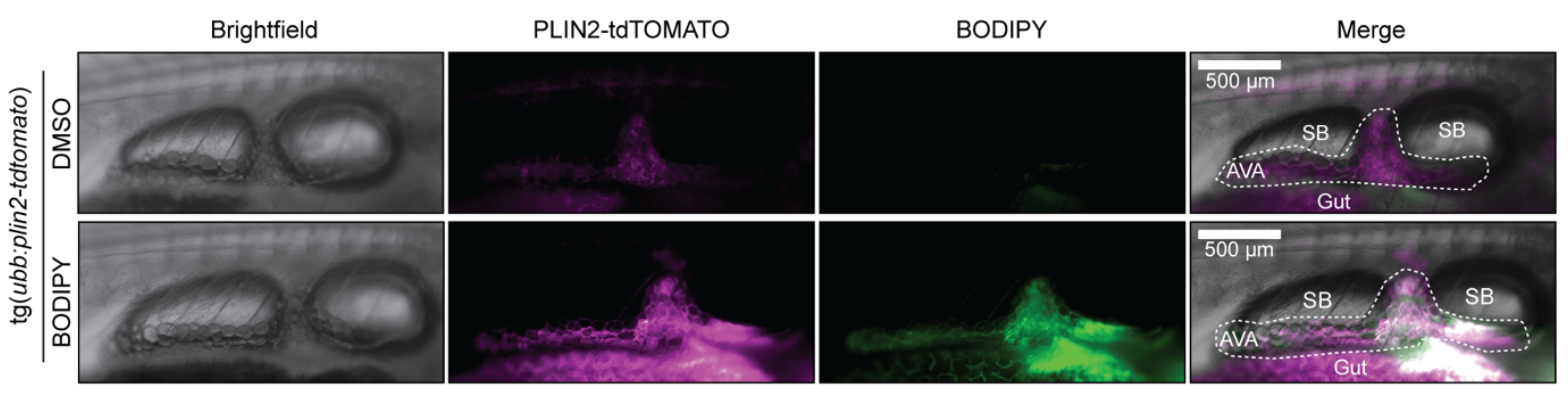

D
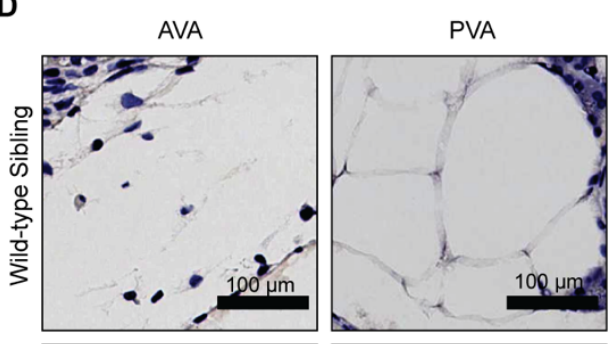

E
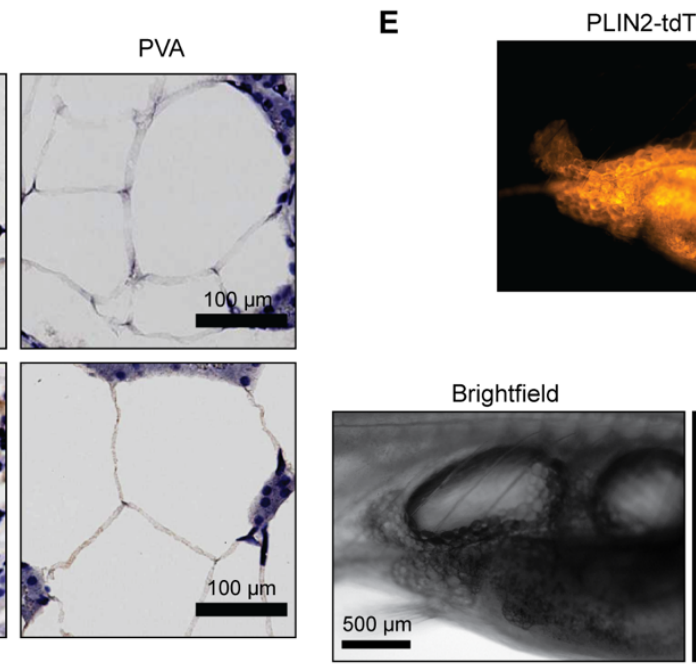

TTOMATO
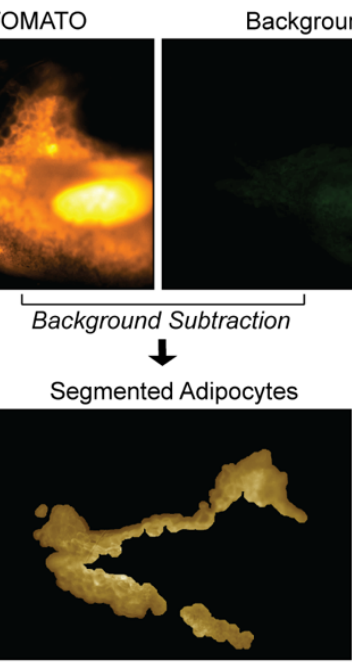

Segmentation Overlay

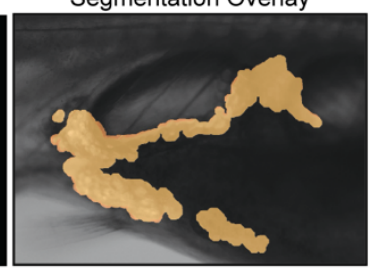

Figure 2: The tg(ubb:plin2-tdtomato) is an in vivo reporter for visceral adipocytes

366

367

368

369

370

371

372

373

374

375

376

377

378

379

(A) Schematic of visceral adipose tissue development in the juvenile zebrafish. Abdominal visceral adipocytes (orange) develop around the swim bladder (gray) and pancreatic visceral adipocytes (red) develop ventrally around the pancreas. (B) Brightfield image of juvenile $\operatorname{tg}$ (ubb:plin2-tdtomato) at $21 \mathrm{dpf}$. Red box indicates position of higher magnification images to visualize abdominal visceral adipocytes. (C) Widefield microscope images of juvenile $\operatorname{tg}$ (ubb:plin2-tdtomato) visceral adipocytes co-stained with DMSO or BODIPY. Panels show brightfield, PLIN2-tdTOMATO, BODIPY, and merge. Visceral adipocytes marked with white dash surrounding the swim bladder (SB) and gut. (D) Juvenile wild-type sibling and tg(ubb:plin2tdtomato) zebrafish were fixed and immunohistochemistry conducted for tdTOMATO expression of abdominal (AVA) and pancreatic (PVA) visceral adipocytes. (E) Representative image of computational segmentation of juvenile tg(ubb:plin2-tdtomato) adipocytes. PLIN2-tdTOMATO was background subtracted with GFP fluorescence. Bottom panels show brightfield, segmented adipocytes, and segmentation overlaid on brightfield. 
A

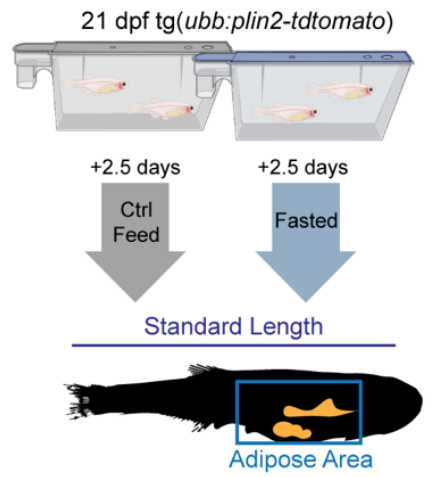

C

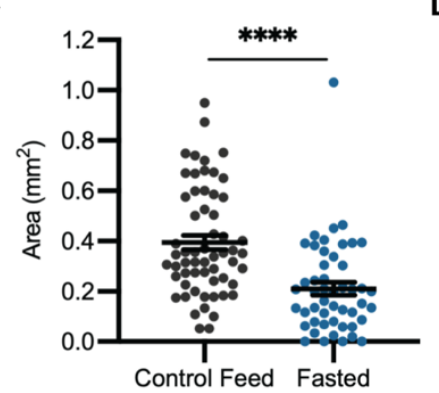

$\mathbf{F}$

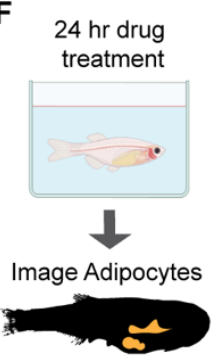

D

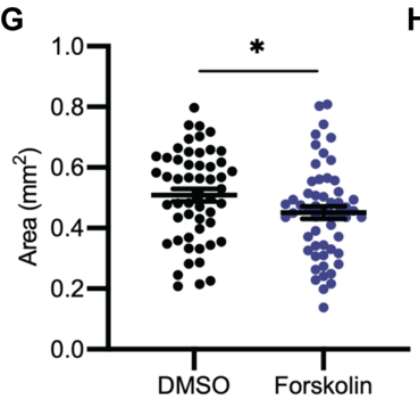

B
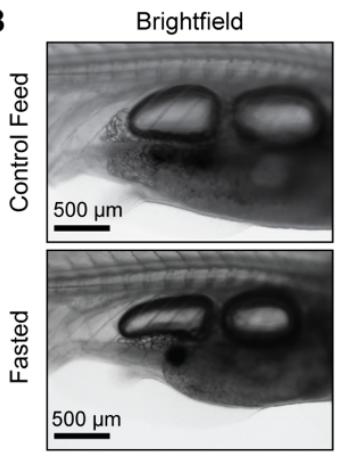

E

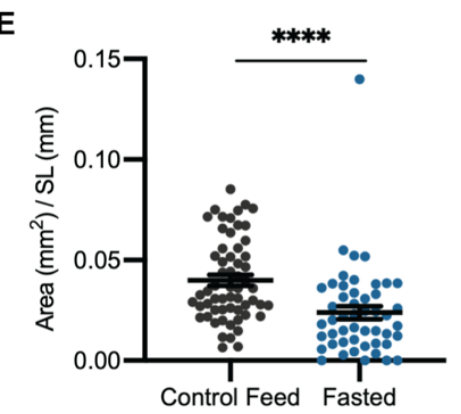

H

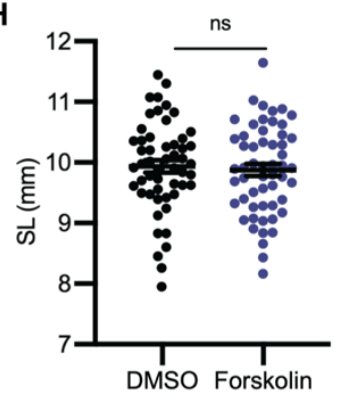

Segmented Adipocytes

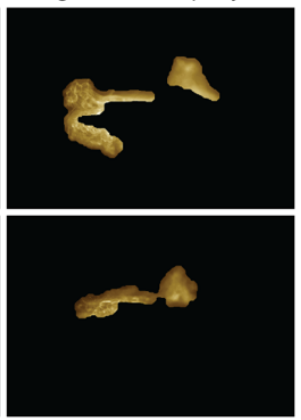

I

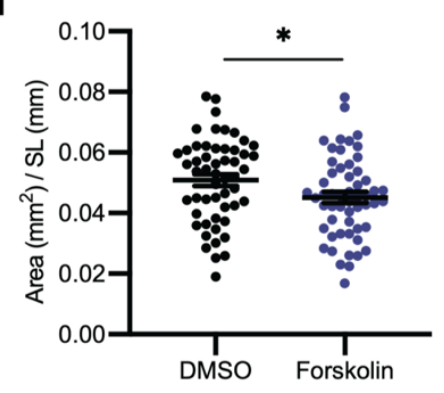

Figure 3: Diet and pharmacologically induced reduction in visceral adipose tissue area

(A) Schematic of experimental set-up for fasting experiment. $21 \mathrm{dpf} \operatorname{tg}$ (ubb:plin2-tdtomato) were fed control feed or fasted for 2.5 days and imaged to measure standard length and adipose area. (B) Representative images of zebrafish given control feed or fasted. Panels show images in brightfield and adipocyte segmentation. Image analysis pipeline resulted in measurements of adipose tissue (C) area, (D) standard length, and (E) area/standard length. Data points indicate individual fish for $N=4$ independent experiments; Control feed $n=49$; Fasted $n=59$. Bars indicate mean and SEM. Significance calculated via Mann-Whitney test; ${ }^{* * * *} p<0.0001$. (F) Schematic of experimental set-up for Forskolin drug treatment. $21 \mathrm{dpf} \operatorname{tg}$ (ubb:plin2-tdtomato) were individually placed in 6 well plates with either DMSO or $5 \mu \mathrm{M}$ Forskolin for 24 hours. Adipose tissue was imaged and analyzed for $(\mathrm{G})$ area, $(\mathrm{H})$ standard length, and $(\mathrm{I})$ area/standard length. Data points indicate individual fish for $\mathrm{N}=5$ independent experiments; DMSO $n=53$; Forskolin $n$ $=55$. Bars indicate mean and SEM. Significance calculated via Mann-Whitney test; ${ }^{*} p<0.05$. 
A $21 \mathrm{dpf} \operatorname{tg}($ ubb:plin2-tdtomato)

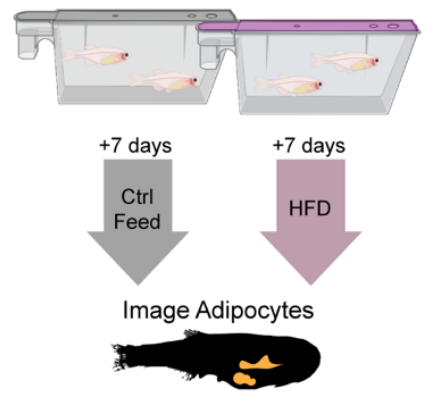

B

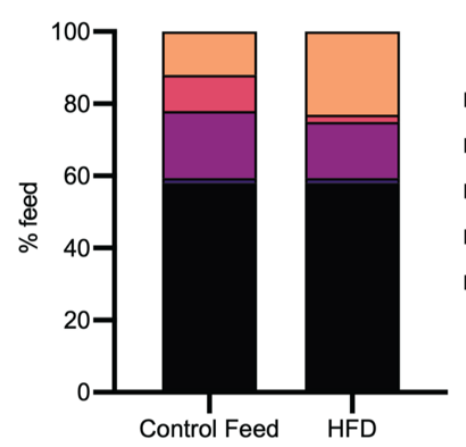

D

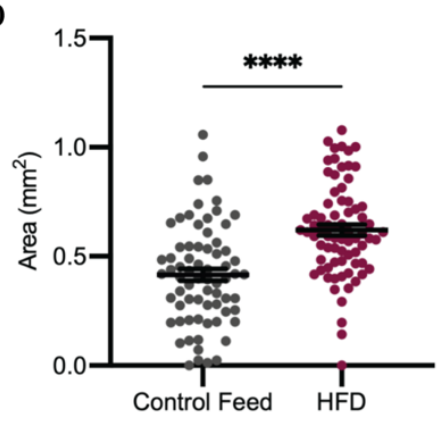

E

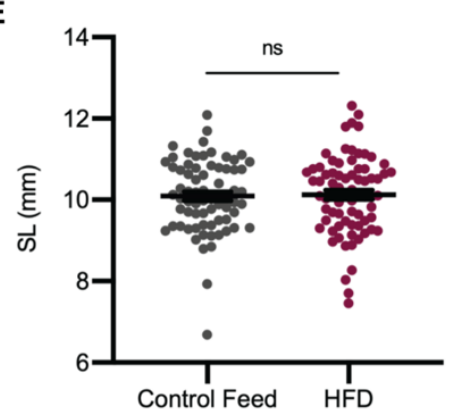

C

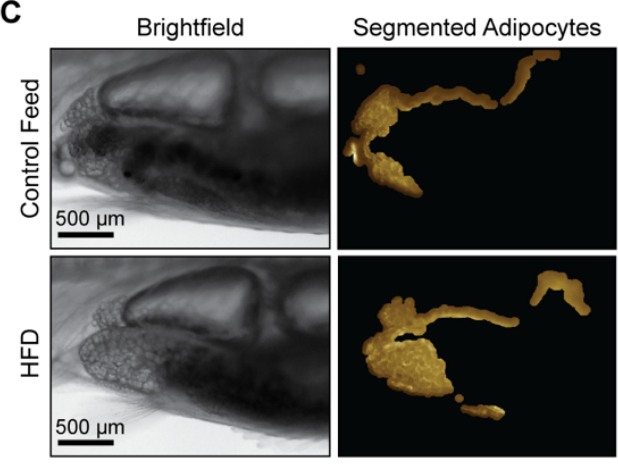

F

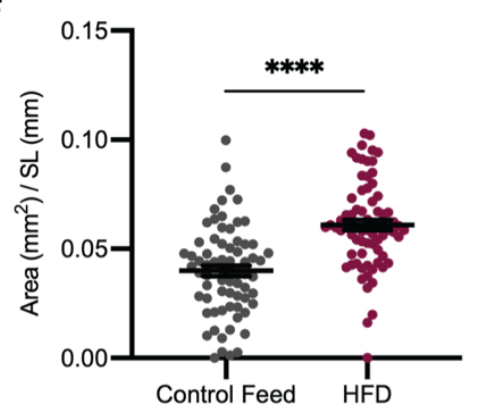

\section{Figure 4: High-fat diet leads to specific enlargement of visceral adipose tissue}

(A) Schematic of experimental set-up for high-fat diet (HFD) experiment. $21 \mathrm{dpf} \operatorname{tg}$ (ubb:plin2tdtomato) were fed control feed or HFD for 7 days and imaged to measure standard length and adipose area. (B) Percent breakdown of nutritional content for control feed and HFD. (C) Representative images of $\operatorname{tg}(u b b$ :plin2-tdtomato) fed either control feed or HFD. Panels show images in brightfield and adipocyte segmentation. Image analysis pipeline resulted in measurements of adipose tissue (D) area, $(E)$ standard length, and (F) area/standard length. Data points indicate individual fish for $\mathrm{N}=3$ independent experiments; Control feed $n=70$; HFD $n=$ 74. Bars indicate mean and SEM. Significance calculated via Mann-Whitney test; ${ }^{* * *} p<0.0001$. 
A

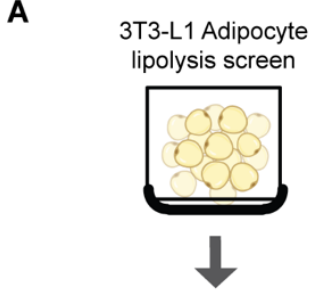

Glycerol Release Assay
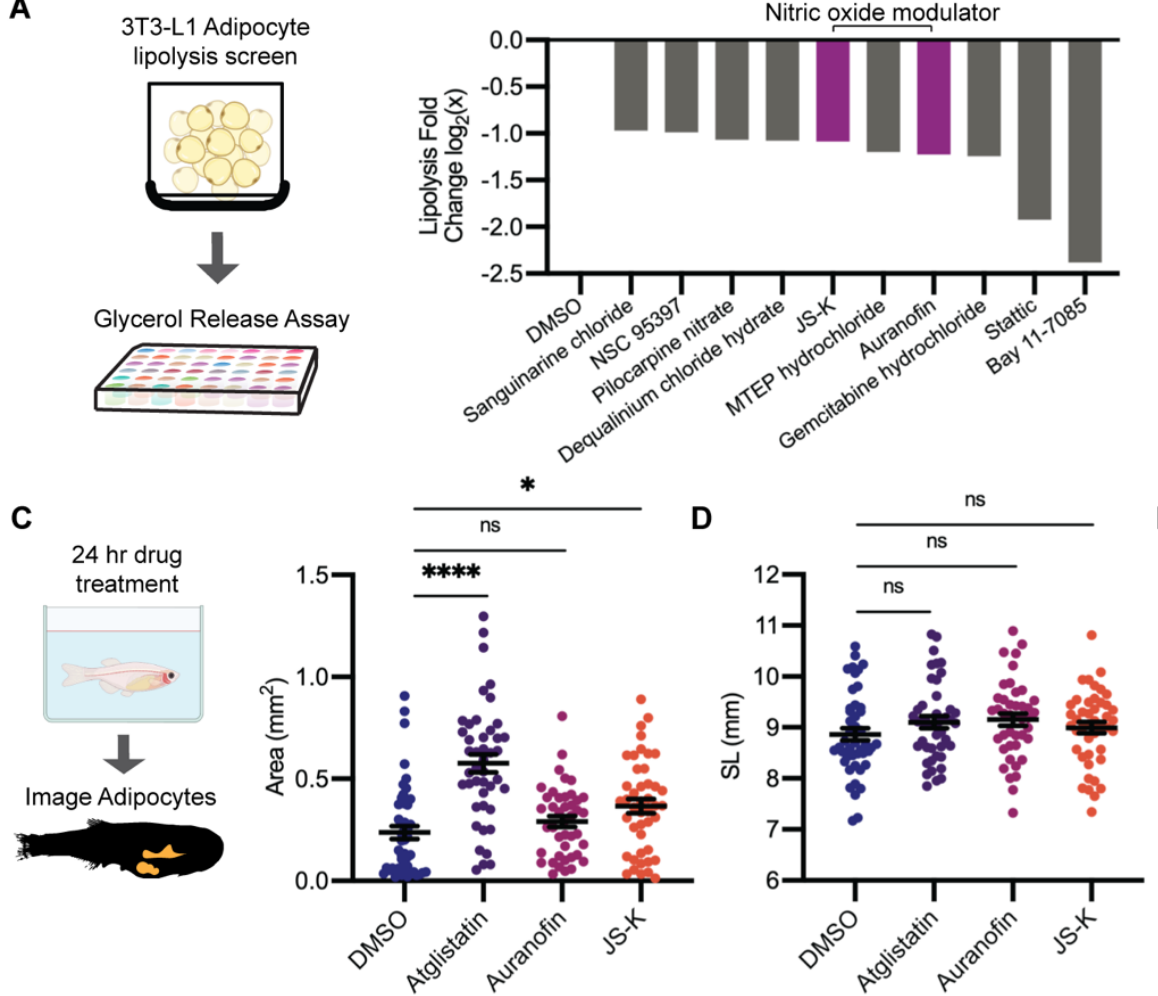

B

E
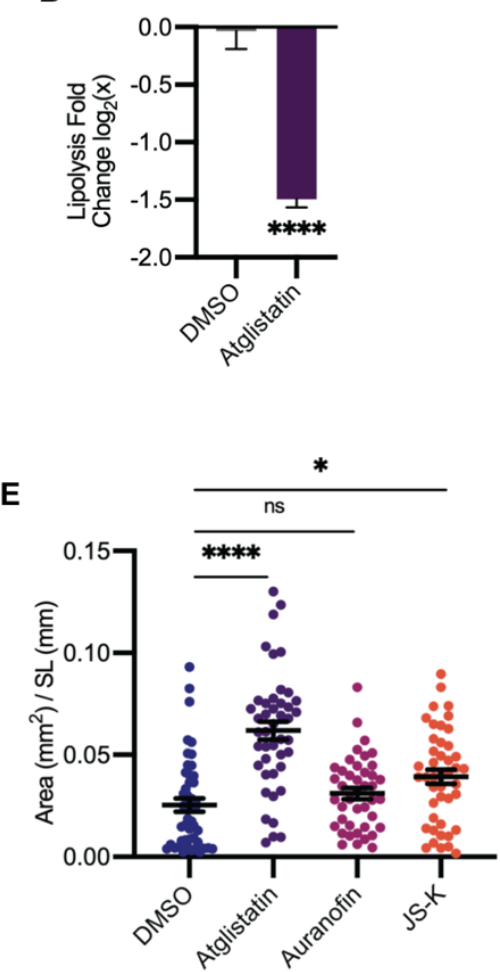

Figure 5: A screen to discover novel compounds that modulate lipolysis and lipid droplets in vivo

(A) Schematic of pharmacologic lipolysis screen in 3T3-L1 adipocytes using a glycerol release assay. Normalized values $\log _{2}$ transformed values for top ten drugs that inhibit lipolysis shown. Magenta indicates compounds that modulate nitric oxide. (B) Normalized values $\log _{2}$ transformed values for lipolysis inhibition in 3T3-L1 adipocytes using either DMSO or $100 \mu \mathrm{M}$ Atglistatin. $\mathrm{N}=$ 5 independent experiments. Bars indicate mean and SEM. Significance calculated via unpaired t-test; ${ }^{* * * *} p<0.0001$. (C) Schematic of experimental set-up for drug treatment. $21 \mathrm{dpf} \operatorname{tg}$ (ubb:plin2tdtomato) were individually placed in 6 well plates with either DMSO, $40 \mu \mathrm{M}$ Atglistatin, $1 \mu \mathrm{M}$ Auranofin, $1 \mu \mathrm{M}$ JS-K for 24 hours. Adipose tissue was imaged and analyzed for (C) area, (D) standard length, and (E) area/standard length. Data points indicate individual fish for $\mathrm{N}=4$ independent experiments; DMSO $n=47$; Atglistatin $n=44$; Auranofin $n=42$; JS-K $n=44$. Bars indicate mean and SEM. Significance calculated via Kruskal-Wallis test; ${ }^{*} p<0.05 ;{ }^{* * *} p<0.0001$. 
A

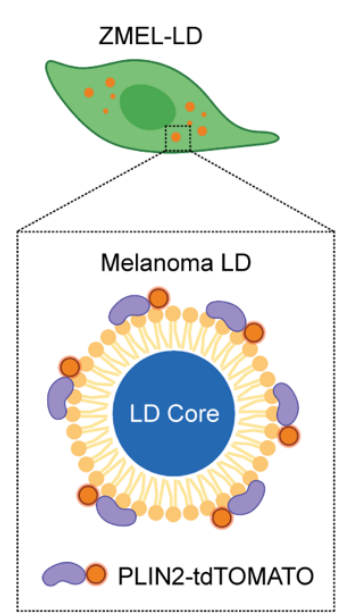

C

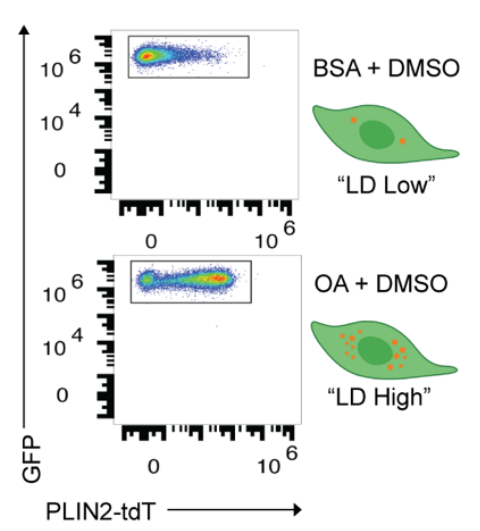

B
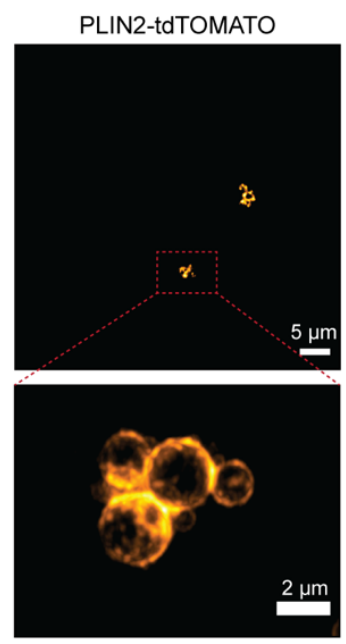

D

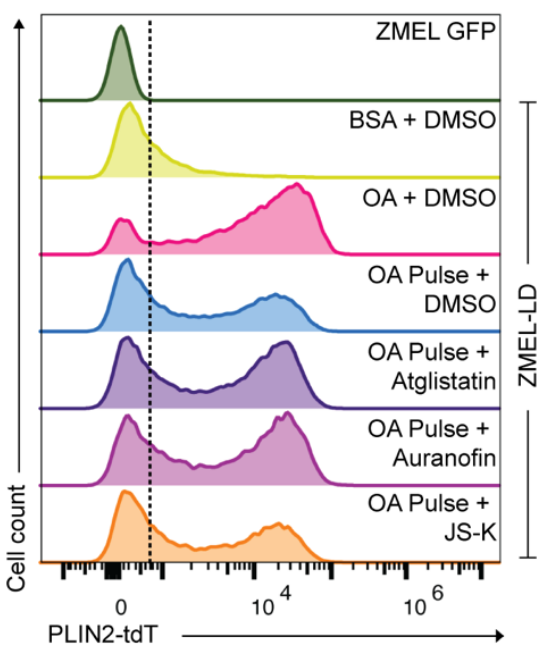

$\mathrm{MDH}$
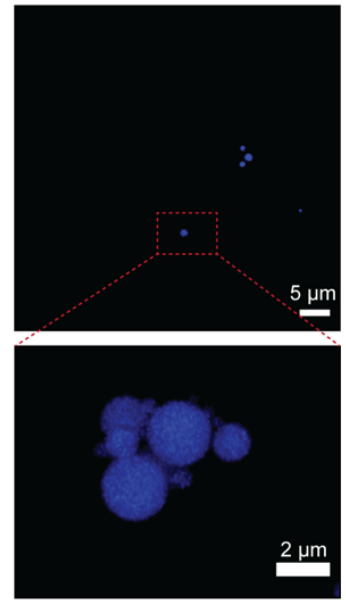

E

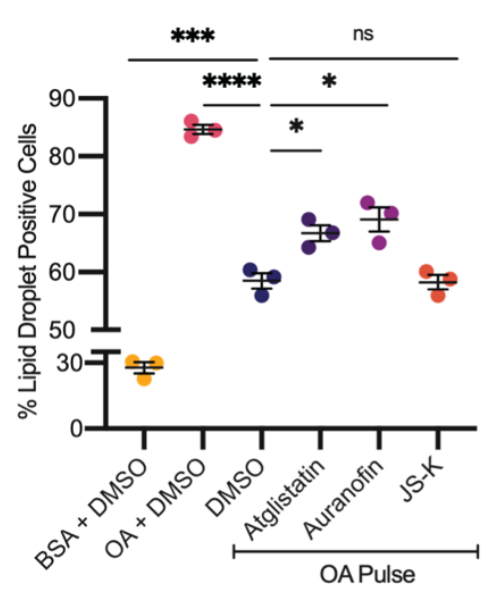

Figure 6: Lipolysis modulators also inhibit lipid droplet loss in melanoma cells

(A) Schematic of zebrafish melanoma cell line with lipid droplet reporter (ZMEL-LD) with lipid droplet labeled by PLIN2-tdTOMATO. (B) Confocal images of ZMEL-LD cells after 24 hours of oleic acid. Panels show fluorescence signal in PLIN2-tdTOMATO, MDH (lipid droplet dye) staining and merge of images with cytoplasmic GFP. Red box indicates position of higher magnification image of lipid droplets. (C) ZMEL-LD cells were treated with either BSA or oleic acid with DMSO for 72 hours then analyzed by FACS for PLIN2-tdTOMATO expression. (D) Representative histogram of PLIN2-tdTOMATO expression of ZMEL GFP and GFP+ ZMEL-LD cells with indicated drugs. Dashed line shows threshold for PLIN2-tdTOMATO expression. (E) Quantification of percent of GFP+ ZMEL-LD cells with lipid droplets. Lipid droplet low and high controls were ZMEL-LD cells treated with BSA or oleic acid for 72 hours. For drug treatments, ZMEL-LD cells were pulsed with oleic acid for 24 hours then given DMSO, $40 \mu \mathrm{M}$ Atglistatin, 0.5 $\mu \mathrm{M}$ Auranofin, or $0.5 \mu \mathrm{M}$ JS-K for 48 hours. $\mathrm{N}=3$ independent experiments. Bars indicate mean and SEM. Significance calculated via unpaired t-test; ${ }^{*} p<0.05 ;{ }^{* * *} p<0.001 ;{ }^{* * *} p<0.0001$. 
bioRxiv preprint doi: https://doi.org/10.1101/2020.11.09.375667; this version posted November 9, 2020. The copyright holder for this preprint (which was not certified by peer review) is the author/funder, who has granted bioRxiv a license to display the preprint in perpetuity. It is made available under aCC-BY-NC 4.0 International license.

\section{Movie 1: 3D Reconstruction of ZMEL-LD Lipid Droplet}

ZMEL-LD cells were given oleic acid for 24 hours, fixed and stained with the lipid droplet dye $\mathrm{MDH}$. This movie is a 3D reconstruction of 37 planes covering a $6 \mu \mathrm{m}$ stack of a lipid droplet 438 cluster in a ZMEL-LD cell. PLIN2-tdTOMATO (orange) is located outside of the lipid droplet core (blue). 


\section{Materials and Methods}

\section{Cloning of ubb:plin2-tdtomato}

To clone the plin2 cDNA, tissue from the muscle and heart of adult casper zebrafish was dissected, pooled and then RNA was isolated using the Zymogen Quick RNA Miniprep Kit (Zymo Research, Irvine, USA, Catalog \#R1054) according to manufacturer instructions. The Invitrogen SuperScriptIII First-Strand Synthesis SuperMix Kit (Thermo Fisher, Waltham, USA Catalog \#18080400) was used according to manufacturer instructions to produce cDNA. CloneAmp HiFi PCR Premix (Takara, Mountain View, USA, Catalog \#639298) was used to PCR amplify the PLIN2 cDNA and gel purified via NucleoSpin Gel and PCR Clean Up (Takara, Mountain View, USA, Catalog \#740609.50). To generate pME-PLIN2-tdTOMATO, the PLIN2 cDNA was inserted on the 5' end of pME-tdTOMATO using In-Fusion HD Cloning Plus (Takara, Mountain View, USA, Catalog \#638920). Gateway cloning using the Gateway LR Clonase Enzyme mix (Thermo Fisher, Waltham, USA Catalog \#11791019) was employed to create the ubb:plin2-tdtomato construct with p5E-ubb, pME-PLIN2-tdTOMATO, p3E-polyA into the pDestTol2pA2-blastocidin (cells) (Heilmann et al., 2015) or pDestTol2CG2 (zebrafish) (Kwan et al., 2007).

\section{Primers}

\begin{tabular}{|c|c|}
\hline \hline Primer name & Primer Sequence \\
\hline Plin2 cDNA Fwd & AAAGCAGGCTCCACCATGAGCTTTCTTCTGTACTTGAAACTG \\
\hline Plin2 cDNA Rev & GCCCTTGCTCACCATTTCAGTGACTTGAAGGGTCCTCTGT \\
\hline Plin2-TMT Fwd & GCCGCCCCCTTCACCATGAGCTTTCTTCTGTACTTGAAAC \\
\hline Plin2-TMT Rev & GCCCTTGCTCACCATTTCAGTGACTTG \\
\hline Tomato ME Plin2 Fwd & ATGGTGAGCAAGGGCGAG \\
\hline Tomato ME Plin 2 Rev & GGTGAAGGGGGCGGC \\
\hline
\end{tabular}

\section{Zebrafish Husbandry}

All zebrafish experiments were carried out in accordance with institutional animal protocols. All zebrafish were housed in a temperature $\left(28.5^{\circ} \mathrm{C}\right.$ ) and light-controlled (14 hours on, 10 hours off) room. Fish were initially housed at a density of 5 fish per liter and fed 3 times per day using rotifers and pelleted zebrafish food. All anesthesia was done using Tricaine (Western Chemical Incorporated, Ferndale, USA) with a stock of $4 \mathrm{~g} / \mathrm{L}$ (protected for light) and diluted until the fish was immobilized. All procedures were approved by and adhered to Institutional Animal Care and Use Committee (IACUC) protocol \#12-05-008 through Memorial Sloan Kettering Cancer Center.

Generation of tg(ubb:plin2-tdtomato)

The ubb:plin2-tdtomato plasmid was injected into casper embryos with Tol2 mRNA to introduce stable integration of the ubb:plin2-tdtomato cassette. Fish with GFP+ hearts (due to pDestTol2CG) were selected and outcrossed to casper fish to produce the F1 generation. F1 zebrafish with GFP+ hearts and validated PLIN2-tdTOMATO expressing adipocytes were outcrossed to generate F2 generation zebrafish for experiments.

\section{Zebrafish Imaging and Analysis} Zebrafish were imaged using an upright Zeiss AxioZoom V16 Fluorescence Stereo Zoom Microscope with a $0.5 x$ (for adult fish) or 1.0x (for juvenile fish) adjustable objective lens equipped with a motorized stage, brightfield, and GFP and tdTomato filter sets. To acquire images, zebrafish were lightly anesthetized with $0.2 \%$ Tricaine. Images were acquired with the 
Zeiss Zen Pro v2 and exported as CZI files for visualization using FIJI or analysis using FIJI (to manually quantify standard length) and MATLAB (Mathworks, Natick, USA).

Our adipocyte segmentation approach utilized the Image Processing Toolbox within MATLAB. Because the zebrafish gut is highly autofluorescent, we chose a threshold for the GFP channel to categorize as background signal and subtracted it from a determined threshold for the tdTOMATO channel. We used a set size to crop images around the tdTOMATO positive signal and created a mask for the adipose tissue. Within the masked area, we applied a higher tdTOMATO threshold to segment the fluorescent signal from the adipocytes. Finally, we quantified the number of pixels above the threshold to quantify adipose tissue area. For visualization purposes, the segmented images were color filtered on Adobe Photoshop from grayscale to gold color scale.

\section{BODIPY staining of zebrafish}

Adult zebrafish were placed in tanks and juvenile zebrafish were placed in p1000 tip boxes with either DMSO or $10 \mathrm{ng} / \mu \mathrm{L}$ BODIPY 493/503 (Thermo Fisher, Waltham, USA, Catalog \#D3922) for 30 mins in the dark. Fish were washed then placed in new tanks with fresh water for 1 hour. Fish were washed again to remove any residual BODIPY then anesthetized and imaged as indicated above for whole adipose tissue.

Higher resolution images of zebrafish adipocytes were acquired using the Zeiss LSM 880 inverted confocal microscope with using a 10x objective. Zebrafish were lightly anesthetized with $0.2 \%$ Tricaine and mounted on a glass bottom dish (MatTek, Ashland, USA, Catalog \#P35G-1.5-20-C) with 0.1\% low gelling agarose (Sigma-Aldrich, St. Louis, USA, Catalog, \#A9045-25G).

\section{IHC for tdTOMATO}

Zebrafish were sacrificed in an ice bath for at least 15 minutes. For adults, zebrafish tails were dissected. For juvenile zebrafish, the entire fish was used for fixation. Selected zebrafish were fixed in $4 \%$ paraformaldehyde for 72 hours at $4^{\circ} \mathrm{C}$, washed in $70 \%$ ethanol for 24 hours, and then paraffin embedded. Fish were sectioned at $5 \mu \mathrm{m}$ and placed on Apex Adhesive slides, baked at $60^{\circ} \mathrm{C}$, and then stained with antibodies against tdTomato (1:500, Rockland, \#600-401379). All histology was performed and stained by Histowiz.

\section{Juvenile Zebrafish Fast} tg(ubb:plin2-tdtomato) F1 fish were outcrossed to caspers to generate the F2 generation. F2 fish were raised at a standard density of 25 fish per $2.8 \mathrm{~L}$ tank. At $21 \mathrm{dpf}$, fish were separated into new tanks which received standard feed or were fasted for 2.5 days. Fish were anesthetized with tricaine and imaged as described above to quantify visceral adipose tissue area and standard length.

\section{High-fat diet feeding} $\operatorname{tg}$ (ubb:plin2-tdtomato) F2 zebrafish were raised at a standard density of 25 fish per $2.8 \mathrm{~L}$ tank. At $21 \mathrm{dpf}$, the zebrafish were placed into $0.8 \mathrm{~L}$ tanks and fed either a high fat or control diet (Sparos, Portugal) for 7 days. Fish were then imaged for Plin2-tdtomato expression at $28 \mathrm{dpf}$. Prior to imaging, fish put in a new tank and food withheld for $\sim 16-20$ hours. Zebrafish were at equal density for control and experimental groups, ranging from 15-30 fish per tank. Fish were fed $0.1 \mathrm{~g}$ feed per tank per day split over two feedings. The high fat and control diets were customized and produced at Sparos Lda (Olhão, Portugal), where powder ingredients were initially mixed according to each target formulation in a double-helix mixer, being thereafter ground twice in a micropulverizer hammer mill (SH1, Hosokawa-Alpine, Germany). The oil 
fraction of the formulation was subsequently added and diets were humidified and agglomerated through low-shear extrusion (Dominioni Group, Italy). Upon extrusion, diets were dried in a convection oven (OP 750-UF, LTE Scientifics, United Kingdom) for $4 \mathrm{~h}$ at $60^{\circ} \mathrm{C}$, being subsequently crumbled (Neuero Farm, Germany) and sieved to 400 microns. Experimental diets were analyzed for proximal composition. The Sparos control diet contains 30\% fishmeal, 33\% squid meal, $5 \%$ fish gelatin, $5.5 \%$ wheat gluten, $12 \%$ cellulose, $2.5 \%$ Soybean oil, $2.5 \%$ rapeseed oil, $2 \%$ vitamins and minerals, $0.1 \%$ vitamin $\mathrm{E}, 0.4 \%$ antioxidant, $2 \%$ monocalcium phosphate, and $2.2 \%$ calcium silicate. The Sparos HFD contains $30 \%$ fishmeal, $33 \%$ squid meal, $5 \%$ fish gelatin, $5.5 \%$ wheat gluten, $12 \%$ palm oil, $2.5 \%$ soybean oil, $2.5 \%$ rapeseed oil, $2 \%$ vitamins and minerals, $0.1 \%$ vitamin $\mathrm{E}, 0.4 \%$ antioxidant, $2 \%$ monocalcium phosphate, and $2.2 \%$ calcium silicate.

\section{T3-L1 Cell Culture}

3T3-L1 cells were acquired from ZenBio and followed their differentiation protocol. Cells are received at Passage 8 and split to a maximum of Passage 12 as per recommendation of the company. 96-well plates were coated with fibronectin (EMD Millipore, Burlington, USA, Catalog \#FC010) diluted 1:100 in PBS for at least 30 minutes to promote improved adherence of cells to the dish. 3T3-L1 cells are first cultured in PM-1-L1 Preadipocyte Medium and allowed to grow to $100 \%$ confluence. PM-1-L1 media is changed every $48-72$ hours. 48 hours after reaching $100 \%$ confluence, cells were changed to DM-1-L1 Differentiation Medium for 72 hours and then changed to AM-1-L1 Adipocyte Media. AM-1-L1 Adipocyte Media was changed every 48-72 hours. Once in AM-1-L1, media is changed gently with a multichannel pipette and only $150 \mu \mathrm{L}$ of the $200 \mu \mathrm{L}$ is replaced to prevent touching the bottom of the well with the pipette tip. After 2-3 weeks in AM-1-L1, the 3T3-L1 develop significantly large lipid droplets and were used in the screen.

\section{LOPAC Library Screen}

The LOPAC library includes 1280 clinically relevant compounds with annotated targets or pathways. The workflow of the screen involved drug or vehicle control of the 3T3-L1 adipocytes for $24 \mathrm{hrs}$ in serum free media. After 24 hours, $100 \mu \mathrm{L}$ of the media supernatant was collected to measure secreted glycerol using the Free Glycerol Reagent (Sigma-Aldrich, St. Louis, USA, Catalog F6428) and following the associated glycerol assay protocol.

Media (screen media) used for drug treatment was phenol-free DMEM supplemented with $0.2 \%$ BSA FFA-free (Sigma-Aldrich, St. Louis, USA, Catalog 9048-46-8). The 1280 compounds were aliquoted as $2 \mu \mathrm{L}$ at $1 \mathrm{mM}$ into $16 \mathrm{x}$ 96-well plates and stored at -20C. Upon thawing, $198 \mu \mathrm{L}$ of screen media was added to the well, bringing the final drug concentration for all compounds in the screen to $10 \mu \mathrm{M}$. Control vehicle was $1 \%$ DMSO served as a negative control and $1 \mathrm{uM}$ Isoproterenol served as a positive control in the screen. This media containing LOPAC drugs, DMSO, and Isoproterenol was transferred to 3T3-L1 cells and incubated for 24 hours.

To measure glycerol release as a readout for lipolysis, $100 \mu \mathrm{L}$ of Free Glycerol Reagent was aliquoted per well of a 96 well plate. $10 \mu \mathrm{L}$ of supernatant media from 3T3-L1 adipocytes was then added to each well. A standard curve was produced by using Glycerol Standard Solution (Sigma-Aldrich, St. Louis, USA, Catalog G7793). The plate is incubated at $37 \mathrm{C}$ for 5 minutes and then developed with a plate reader set to detect absorbance at $540 \mathrm{~nm}$. Using the standard curve, a fit equation is developed in Excel to convert the absorbance values into glycerol concentration. To take into account differences that occur in wells on the edge versus middle of the plate, all well positions across all plates in the screen are averaged to create a normalization factor for any given position on the plate. These normalized values were then used to determine top hits for compounds either that block lipolysis. 
584

585

586

587

588

589

590

591

592

593

594

595

596

597

598

599

600

601

602

603

604

605

606

607

608

609

610

611

612

613

614

615

616

617

618

619

620

621

622

623

624

Glycerol Release Assay with Atglistatin

3T3-L1s were differentiated on a fibronectin-coated 96-well dish. At the start of the lipolysis experiment, 3T3-L1s were changed to serum-free DMEM supplemented with $0.2 \%$ BSA FFAfree (Sigma-Aldrich, St. Louis, USA, Catalog 9048-46-8). The media was supplemented with $1 \%$ DMSO for negative control or $1 \mathrm{uM}$ isoproterenol to induce lipolysis +/- $100 \mu \mathrm{M}$ Atglistatin (Sigma-Aldrich, St. Louis, USA, Catalog SML1075) to block lipolysis and cells were incubated for 24 hours.

To measure glycerol release, $100 \mu \mathrm{L}$ of Free Glycerol Reagent was aliquoted per well of a new 96 well plate. $10 \mu \mathrm{L}$ of supernatant media from 3T3-L1 adipocytes was then added to each well. A standard curve was produced by using Glycerol Standard Solution (Sigma-Aldrich, St. Louis, USA, Catalog G7793). The plate is incubated at $37 \mathrm{C}$ for 5 minutes and then developed with a plate reader set to detect absorbance at $540 \mathrm{~nm}$. Using the standard curve, a fit equation is developed in Excel to convert the absorbance values into glycerol concentration.

Juvenile Zebrafish Drug Treatments tg(ubb:plin2-tdtomato) F1 fish were outcrossed to caspers to generate the F2 generation. F2 fish were raised at a standard density of 50 fish per $6.0 \mathrm{~L}$ tank. For drug treatment, fish were removed from the system at $21 \mathrm{dpf}$ and placed at a density of 1 fish per well in a 6 well plate with $10 \mathrm{~mL}$ of E3 per well. After a 24 hour incubation with the drug fish were anesthetized with Tricaine and imaged using the described protocol to quantify (1) Standard length and (2) area of PLIN2-tdTOMATO expression corresponding to visceral adipose tissue area. Fish were treated with the following compounds, which were all dissolved in DMSO.

\begin{tabular}{|c|c|c|}
\hline Compound & Dose & Source \\
\hline Forskolin & $5 \mu \mathrm{M}$ & $\begin{array}{c}\text { Sigma-Aldrich, St. Louis, USA, } \\
\text { Catalog, Catalog \#F6886 }\end{array}$ \\
\hline Auranofin & $1 \mu \mathrm{M}$ & $\begin{array}{c}\text { Sigma-Aldrich, St. Louis, USA, } \\
\text { Catalog, Catalog \#A6733 }\end{array}$ \\
\hline JS-K & $1 \mu \mathrm{M}$ & $\begin{array}{c}\text { Sigma-Aldrich, St. Louis, USA, } \\
\text { Catalog, Catalog \#J4137 }\end{array}$ \\
\hline Atglistatin & $40 \mu \mathrm{M}$ & $\begin{array}{c}\text { Sigma-Aldrich, St. Louis, USA, } \\
\text { Catalog, Catalog \#SML1075 }\end{array}$ \\
\hline
\end{tabular}

Generation of ZMEL-LD Cell Line

The ZMEL zebrafish melanoma cell line was derived from a tumor of a mitfa:BRAF $F^{\mathrm{V} 600 \mathrm{E}} / \mathrm{p} 53^{-/-}$ zebrafish was described previously (Heilmann et al., 2015). ZMEL cells constitutively express eGFP driven by the mitfa promoter(Heilmann et al., 2015). ZMEL cells were grown at $28^{\circ} \mathrm{C}$ in a humidified incubator in DMEM (Gibco, Waltham, USA, Catalog, \#11965) supplemented with 10\% FBS (Gemini Bio, \#100-500), 1X penicillin/streptomycin/glutamine (Gibco, Waltham, USA, Catalog, \#10378016), and 1X GlutaMAX (Gibco, Waltham, USA, Catalog, \#35050061). To generate the ZMEL-LD cells, ZMEL cells were nucleofected with the ubb:plin2-tdtomato plasmid using the Neon Transfection System (Thermo Fisher, Waltham, USA, Catalog \#MPK10096), selected for two weeks in blasticidine supplemented media at $4 \mu \mathrm{g} / \mu \mathrm{L}$ (Sigma-Aldrich, St. Louis, USA, Catalog, \#15205-25MG), and FACS sorted for GFP and tdTOMATO double positive cells.

ZMEL-LD Imaging

8 well Nunc Lab-Tek Chambered Coverglass was coated with 1:100 dilution of fibronectin in DPBS (Millipore Sigma, Burlington, USA, Catalog \#FC010-5MG) for 30 mins and then washed 
with DPBS (Thermo Scientific, Waltham, USA, Catalog, \#14190-250). ZMEL-LD cells were seeded at 30,000 cells per well and left to adhere for 24 hours. Media supplemented with 250 $\mu \mathrm{M}$ oleic acid (Sigma-Aldrich, St. Louis, USA, Catalog, \#O3008-5ML) was added for 24 hours. Cells were fixed with $2 \%$ paraformaldehyde (Santa Cruz Biotechnology, Santa Cruz, USA, Catalog \#sc-281692) for 45 minutes, washed with DPBS and permeabilized with $0.1 \%$ triton-X (Thermo Fisher, Waltham, USA, Catalog \#PI85111) for 30 minutes at room temperature. To stain for lipid droplets, cells were washed and stained with 1:500 MDH (Abcepta, San Diego, USA, Catalog \#SM1000a) for 15 minutes. Cells were imaged on the Zeiss LSM 880 inverted confocal microscope with AiryScan using a 63x oil immersion objective. Confocal stacks were visualized via FIJI and 3D reconstruction was created using Imaris (Bitplane Inc, Concord, USA).

\section{ZMEL-LD FACS Analysis}

ZMEL Dark (no fluorescence), ZMEL-GFP, ZMEL-LD cells were plated on fibronectin coated 6 well plates at a density of 500,000 cells in $1 \mathrm{~mL}$ of media per well. At 24 hours after plating, cells were given either $150 \mu \mathrm{M}$ of BSA or oleic acid with $1 \mu \mathrm{L}$ of DMSO. At 48 and 72 hours after plating, lipid droplet low and high controls were switched to fresh media with $150 \mu \mathrm{M}$ of BSA or oleic acid with $1 \mu \mathrm{L}$ of DMSO. Cells pulsed with oleic acid received fresh media with 150150 $\mu \mathrm{M}$ of BSA with either $40 \mu \mathrm{M}$ Atglistatin, $0.5 \mu \mathrm{M}$ Auranofin or $0.5 \mu \mathrm{M}$ JS-K. At 96 hours after plating, cells were trypsinized, washed with DPBS and resuspended in DMEM supplemented with $2 \%$ FBS, 1X penicillin/streptomycin/glutamine, and 1X GlutaMAX. Cells were stained for viability with 1:1000 DAPI and strained through the Falcon FACS Tube with Cell Strainer Cap (Thermo Fisher, Waltham, USA Catalog, \#08-771-23). Data was acquired via the Beckman Coulter CytoFLEX Flow Cytometer (Beckman Coulter, Miami, USA) and analyzed via FlowJo software (BD Biosciences, San Jose, USA).

\section{Schematics}

Schematics and illustrations were generated via Biorender on biorender.com.

\section{Statistics}

All statistical analysis was performed using GraphPad Prism 8 (Graphpad, San Diego, USA). Data are presented as mean \pm standard error (SEM). $\mathrm{P}<0.05$ was considered statistically significant. Statistical tests used were Mann-Whitney, Kruskal-Wallis or unpaired t-tests which are noted in the figure legend. All experiments were done with at least 3 independent replicates. For in vivo experiments, $\mathrm{N}$ denotes number of independent experiments while $\mathrm{n}$ denotes number of individual fish. Imaging analysis utilized FIJI, Imaris, MATLAB software. 
661

662

663

664

665

666

667

668

669

670

671

672

673

674

675

676

677

678

679

680

681

682

683

684

685

686

687

688

689

690

691

692

693

694

695

696

697

698

699

700

701

702

703

704

705

706

707

708

709

710

\section{References}

Ahmadian, M., Abbott, Marcia J., Tang, T., Hudak, Carolyn S. S., Kim, Y., Bruss, M., Hellerstein, Marc K., Lee, H.-Y., Samuel, Varman T., Shulman, Gerald I., Wang, Y., Duncan, Robin E., Kang, C., \& Sul, Hei S. (2011). Desnutrin/ATGL Is Regulated by AMPK and Is Required for a Brown Adipose Phenotype. Cell Metabolism, 13(6), 739-748. doi:https://doi.org/10.1016/i.cmet.2011.05.002

Bailey, Andrew P., Koster, G., Guillermier, C., Hirst, Elizabeth M. A., MacRae, James I., Lechene, Claude P., Postle, Anthony D., \& Gould, Alex P. (2015). Antioxidant Role for Lipid Droplets in a Stem Cell Niche of Drosophila. Cell, 163(2), 340-353. doi:https://doi.org/10.1016/i.cell.2015.09.020

Balaban, S., Shearer, R. F., Lee, L. S., van Geldermalsen, M., Schreuder, M., Shtein, H. C., Cairns, R., Thomas, K. C., Fazakerley, D. J., Grewal, T., Holst, J., Saunders, D. N., \& Hoy, A. J. (2017). Adipocyte lipolysis links obesity to breast cancer growth: adipocytederived fatty acids drive breast cancer cell proliferation and migration. Cancer \& Metabolism, 5(1), 1. doi:10.1186/s40170-016-0163-7

Barba, G., Harper, F., Harada, T., Kohara, M., Goulinet, S., Matsuura, Y., Eder, G., Schaff, Z., Chapman, M. J., Miyamura, T., \& Bréchot, C. (1997). Hepatitis C virus core protein shows a cytoplasmic localization and associates to cellular lipid storage droplets. Proceedings of the National Academy of Sciences, 94(4), 1200. doi:10.1073/pnas.94.4.1200

Bensaad, K., Favaro, E., Lewis, Caroline A., Peck, B., Lord, S., Collins, Jennifer M., Pinnick, Katherine E., Wigfield, S., Buffa, Francesca M., Li, J.-L., Zhang, Q., Wakelam, Michael J. O., Karpe, F., Schulze, A., \& Harris, Adrian L. (2014). Fatty Acid Uptake and Lipid Storage Induced by HIF-1a Contribute to Cell Growth and Survival after HypoxiaReoxygenation. Cell Reports, 9(1), 349-365. doi:https://doi.org/10.1016/j.celrep.2014.08.056

Berry, R., Church, C. D., Gericke, M. T., Jeffery, E., Colman, L., \& Rodeheffer, M. S. (2014). Chapter Four - Imaging of Adipose Tissue. In O. A. Macdougald (Ed.), Methods in Enzymology (Vol. 537, pp. 47-73): Academic Press.

Berry, R., \& Rodeheffer, M. S. (2013). Characterization of the adipocyte cellular lineage in vivo. Nature Cell Biology, 15(3), 302-308. doi:10.1038/ncb2696

Bosch, M., Sánchez-Álvarez, M., Fajardo, A., Kapetanovic, R., Steiner, B., Dutra, F., Moreira, L., López, J. A., Campo, R., Marí, M., Morales-Paytuví, F., Tort, O., Gubern, A., Templin, R. M., Curson, J. E. B., Martel, N., Català, C., Lozano, F., Tebar, F., Enrich, C., Vázquez, J., Del Pozo, M. A., Sweet, M. J., Bozza, P. T., Gross, S. P., Parton, R. G., \& Pol, A. (2020). Mammalian lipid droplets are innate immune hubs integrating cell metabolism and host defense. Science, 370(6514), eaay8085. doi:10.1126/science.aay8085

Bosma, M. (2016). Lipid droplet dynamics in skeletal muscle. Experimental Cell Research, 340(2), 180-186. doi:https://doi.org/10.1016/j.yexcr.2015.10.023

Cabodevilla, A. G., Sánchez-Caballero, L., Nintou, E., Boiadjieva, V. G., Picatoste, F., Gubern, A., \& Claro, E. (2013). Cell survival during complete nutrient deprivation depends on lipid droplet-fueled $\beta$-oxidation of fatty acids. The Journal of biological chemistry, 288(39), 27777-27788. doi:10.1074/jbc.M113.466656

Chi, J., Crane, A., Wu, Z., \& Cohen, P. (2018). Adipo-Clear: A Tissue Clearing Method for Three-Dimensional Imaging of Adipose Tissue. Journal of visualized experiments : JoVE(137), 58271. doi:10.3791/58271

Chitraju, C., Mejhert, N., Haas, J. T., Diaz-Ramirez, L. G., Grueter, C. A., Imbriglio, J. E., Pinto, S., Koliwad, S. K., Walther, T. C., \& Farese, R. V., Jr. (2017). Triglyceride Synthesis by 
DGAT1 Protects Adipocytes from Lipid-Induced ER Stress during Lipolysis. Cell Metabolism, 26(2), 407-418.e403. doi:10.1016/j.cmet.2017.07.012

Chu, C.-Y., Chen, C.-F., Rajendran, R. S., Shen, C.-N., Chen, T.-H., Yen, C.-C., Chuang, C.-K., Lin, D.-S., \& Hsiao, C.-D. (2012). Overexpression of Akt1 Enhances Adipogenesis and Leads to Lipoma Formation in Zebrafish. PLoS One, 7(5), e36474. doi:10.1371/journal.pone.0036474

Duncan, R. E., Ahmadian, M., Jaworski, K., Sarkadi-Nagy, E., \& Sul, H. S. (2007). Regulation of lipolysis in adipocytes. Annual review of nutrition, 27, 79-101. doi:10.1146/annurev.nutr.27.061406.093734

Fam, T. K., Klymchenko, A. S., \& Collot, M. (2018). Recent Advances in Fluorescent Probes for Lipid Droplets. Materials (Basel, Switzerland), 11(9), 1768. doi:10.3390/ma11091768

Farese, R. V., Jr., \& Walther, T. C. (2009). Lipid Droplets Finally Get a Little R-E-S-P-E-C-T. Cell, 139(5), 855-860. doi:10.1016/j.cell.2009.11.005

Fischer, J., Lefèvre, C., Morava, E., Mussini, J.-M., Laforêt, P., Negre-Salvayre, A., Lathrop, M., \& Salvayre, R. (2007). The gene encoding adipose triglyceride lipase (PNPLA2) is mutated in neutral lipid storage disease with myopathy. Nat Genet, 39(1), 28-30. doi:10.1038/ng1951

Fox, C. S., Massaro, J. M., Hoffmann, U., Pou, K. M., Maurovich-Horvat, P., Liu, C.-Y., Vasan, R. S., Murabito, J. M., Meigs, J. B., Cupples, L. A., D’Agostino, R. B., \& O’Donnell, C. J. (2007). Abdominal Visceral and Subcutaneous Adipose Tissue Compartments. Circulation, 116(1), 39-48. doi:doi:10.1161/CIRCULATIONAHA.106.675355

Fujimoto, M., Matsuzaki, I., Nishitsuji, K., Yamamoto, Y., Murakami, D., Yoshikawa, T., Fukui, A., Mori, Y., Nishino, M., Takahashi, Y., Iwahashi, Y., Warigaya, K., Kojima, F., Jinnin, M., \& Murata, S.-i. (2020). Adipophilin expression in cutaneous malignant melanoma is associated with high proliferation and poor clinical prognosis. Laboratory Investigation, 100(5), 727-737. doi:10.1038/s41374-019-0358-y

Fujimoto, T., \& Parton, R. G. (2011). Not just fat: the structure and function of the lipid droplet. Cold Spring Harbor perspectives in biology, 3(3), a004838. doi:10.1101/cshperspect.a004838

Haemmerle, G., Lass, A., Zimmermann, R., Gorkiewicz, G., Meyer, C., Rozman, J., Heldmaier, G., Maier, R., Theussl, C., Eder, S., Kratky, D., Wagner, E. F., Klingenspor, M., Hoefler, G., \& Zechner, R. (2006). Defective Lipolysis and Altered Energy Metabolism in Mice Lacking Adipose Triglyceride Lipase. Science, 312, 734-737.

Heid, H., Rickelt, S., Zimbelmann, R., Winter, S., Schumacher, H., Dörflinger, Y., Kuhn, C., \& Franke, W. W. (2014). On the Formation of Lipid Droplets in Human Adipocytes: The Organization of the Perilipin-Vimentin Cortex. PLoS One, 9(2), e90386. doi:10.1371/journal.pone.0090386

Heilmann, S., Ratnakumar, K., Langdon, E. M., Kansler, E. R., Kim, I. S., Campbell, N. R., Perry, E. B., McMahon, A. J., Kaufman, C. K., van Rooijen, E., Lee, W., lacobuzioDonahue, C. A., Hynes, R. O., Zon, L. I., Xavier, J. B., \& White, R. M. (2015). A Quantitative System for Studying Metastasis Using Transparent Zebrafish. Cancer Research, 75(20), 4272. doi:10.1158/0008-5472.CAN-14-3319

Hellmér, J., Arner, P., \& Lundin, A. (1989). Automatic luminometric kinetic assay of glycerol for lipolysis studies. Analytical Biochemistry, 177(1), 132-137. doi:https://doi.org/10.1016/0003-2697(89)90027-4

Henne, W. M., Reese, M. L., \& Goodman, J. M. (2018). The assembly of lipid droplets and their roles in challenged cells. The EMBO journal, 37(12), e98947. doi:10.15252/embj.201898947

Jarc, E., \& Petan, T. (2019). Lipid Droplets and the Management of Cellular Stress. The Yale journal of biology and medicine, 92(3), 435-452. Retrieved from https://pubmed.ncbi.nlm.nih.gov/31543707 
762

763

764

765

766

767

768

769

770

771

772

773

774

775

776

777

778

779

780

781

782

783

784

785

786

787

788

789

790

791

792

793

794

795

796

797

798

799

800

801

802

803

804

805

806

807

808

809

810

811

812

https://www.ncbi.nlm.nih.gov/pmc/articles/PMC6747940/

Krahmer, N., Farese, R. V., Jr., \& Walther, T. C. (2013). Balancing the fat: lipid droplets and human disease. EMBO molecular medicine, 5(7), 973-983. doi:10.1002/emmm.201100671

Kühnlein, R. P. (2011). The contribution of the Drosophila model to lipid droplet research. Progress in Lipid Research, 50(4), 348-356. doi:https://doi.org/10.1016/i.plipres.2011.04.001

Kuniyoshi, S., Miki, Y., Sasaki, A., Iwabuchi, E., Ono, K., Onodera, Y., Hirakawa, H., Ishida, T., Yoshimi, N., \& Sasano, H. (2019). The significance of lipid accumulation in breast carcinoma cells through perilipin 2 and its clinicopathological significance. Pathology International, 69(8), 463-471. doi:10.1111/pin.12831

Kwan, K. M., Fujimoto, E., Grabher, C., Mangum, B. D., Hardy, M. E., Campbell, D. S., Parant, J. M., Yost, H. J., Kanki, J. P., \& Chien, C.-B. (2007). The Tol2kit: A multisite gatewaybased construction kit for Tol2 transposon transgenesis constructs. Developmental Dynamics, 236(11), 3088-3099. doi:10.1002/dvdy.21343

Landgraf, K., Schuster, S., Meusel, A., Garten, A., Riemer, T., Schleinitz, D., Kiess, W., \& Körner, A. (2017). Short-term overfeeding of zebrafish with normal or high-fat diet as a model for the development of metabolically healthy versus unhealthy obesity. BMC Physiology, 17(1), 4-4. doi:10.1186/s12899-017-0031-x

Le Jemtel, T. H., Samson, R., Milligan, G., Jaiswal, A., \& Oparil, S. (2018). Visceral Adipose Tissue Accumulation and Residual Cardiovascular Risk. Current Hypertension Reports, 20(9), 77. doi:10.1007/s11906-018-0880-0

Lengyel, E., Makowski, L., DiGiovanni, J., \& Kolonin, M. G. (2018). Cancer as a Matter of Fat: The Crosstalk between Adipose Tissue and Tumors. Trends Cancer, 4(5), 374-384. doi:10.1016/j.trecan.2018.03.004

Litosch, I., Hudson, T. H., Mills, I., Li, S. Y., \& Fain, J. N. (1982). Forskolin as an activator of cyclic AMP accumulation and lipolysis in rat adipocytes. Molecular Pharmacology, 22(1), 109-115. Retrieved from https://molpharm.aspetjournals.org/content/molpharm/22/1/109.full.pdf

Liu, Z., Li, X., Ge, Q., Ding, M., \& Huang, X. (2014). A Lipid Droplet-Associated GFP ReporterBased Screen Identifies New Fat Storage Regulators in C. elegans. Journal of Genetics and Genomics, 41(5), 305-313. doi:https://doi.org/10.1016/j.jgg.2014.03.002

Longo, Valter D., \& Mattson, Mark P. (2014). Fasting: Molecular Mechanisms and Clinical Applications. Cell Metabolism, 19(2), 181-192. doi:https://doi.org/10.1016/j.cmet.2013.12.008

Mayer, N., Schweiger, M., Romauch, M., Grabner, G. F., Eichmann, T. O., Fuchs, E., Ivkovic, J., Heier, C., Mrak, I., Lass, A., Höfler, G., Fledelius, C., Zechner, R., Zimmermann, R., \& Breinbauer, R. (2013). Development of small-molecule inhibitors targeting adipose triglyceride lipase. Nature Chemical Biology, 9(12), 785-787. doi:10.1038/nchembio.1359

Minchin, J. E. N., \& Rawls, J. F. (2017). A classification system for zebrafish adipose tissues. Disease Models \&amp;amp; Mechanisms, 10(6), 797. doi:10.1242/dmm.025759

Miyanari, Y., Atsuzawa, K., Usuda, N., Watashi, K., Hishiki, T., Zayas, M., Bartenschlager, R., Wakita, T., Hijikata, M., \& Shimotohno, K. (2007). The lipid droplet is an important organelle for hepatitis C virus production. Nature Cell Biology, 9(9), 1089-1097. doi:10.1038/ncb1631

Nath, N., Chattopadhyay, M., Pospishil, L., Cieciura, L. Z., Goswami, S., Kodela, R., Saavedra, J. E., Keefer, L. K., \& Kashfi, K. (2010). JS-K, a nitric oxide-releasing prodrug, modulates B-catenin/TCF signaling in leukemic Jurkat cells: evidence of an S-nitrosylated mechanism. Biochemical pharmacology, 80(11), 1641-1649.

doi:10.1016/j.bcp.2010.08.011 
813

814

815

816

817

818

819

820

821

822

823

824

825

826

827

828

829

830

831

832

833

834

835

836

837

838

839

840

841

842

843

844

845

846

847

848

849

850

851

852

853

854

855

856

857

858

859

860

861

862

Nguyen, T. B., Louie, S. M., Daniele, J. R., Tran, Q., Dillin, A., Zoncu, R., Nomura, D. K., \& Olzmann, J. A. (2017). DGAT1-Dependent Lipid Droplet Biogenesis Protects Mitochondrial Function during Starvation-Induced Autophagy. Developmental Cell, 42(1), 9-21.e25. doi:10.1016/j.devcel.2017.06.003

Nieman, K. M., Kenny, H. A., Penicka, C. V., Ladanyi, A., Buell-Gutbrod, R., Zillhardt, M. R., Romero, I. L., Carey, M. S., Mills, G. B., Hotamisligil, G. S., Yamada, S. D., Peter, M. E., Gwin, K., \& Lengyel, E. (2011). Adipocytes promote ovarian cancer metastasis and provide energy for rapid tumor growth. Nat Med, 17(11), 1498-1503. doi:10.1038/nm.2492

Oka, T., Nishimura, Y., Zang, L., Hirano, M., Shimada, Y., Wang, Z., Umemoto, N., Kuroyanagi, J., Nishimura, N., \& Tanaka, T. (2010). Diet-induced obesity in zebrafish shares common pathophysiological pathways with mammalian obesity. BMC Physiology, 10(1), 21. doi:10.1186/1472-6793-10-21

Olzmann, J. A., \& Carvalho, P. (2019). Dynamics and functions of lipid droplets. Nature Reviews Molecular Cell Biology, 20(3), 137-155. doi:10.1038/s41580-018-0085-z

Paar, M., Jüngst, C., Steiner, N. A., Magnes, C., Sinner, F., Kolb, D., Lass, A., Zimmermann, R., Zumbusch, A., Kohlwein, S. D., \& Wolinski, H. (2012). Remodeling of lipid droplets during lipolysis and growth in adipocytes. The Journal of biological chemistry, 287(14), 11164-11173. doi:10.1074/jbc.M111.316794

Petan, T., Jarc, E., \& Jusović, M. (2018). Lipid Droplets in Cancer: Guardians of Fat in a Stressful World. Molecules (Basel, Switzerland), 23(8), 1941. doi:10.3390/molecules23081941

Rambold, A. S., Cohen, S., \& Lippincott-Schwartz, J. (2015). Fatty acid trafficking in starved cells: regulation by lipid droplet lipolysis, autophagy, and mitochondrial fusion dynamics. Developmental Cell, 32(6), 678-692. doi:10.1016/j.devcel.2015.01.029

Rosen, Evan D., \& Spiegelman, Bruce M. (2014). What We Talk About When We Talk About Fat. Cell, 156(1), 20-44. doi:10.1016/j.cell.2013.12.012

Schoiswohl, G., Schweiger, M., Schreiber, R., Gorkiewicz, G., Preiss-Landl, K., Taschler, U., Zierler, K. A., Radner, F. P. W., Eichmann, T. O., Kienesberger, P. C., Eder, S., Lass, A., Haemmerle, G., Alsted, T. J., Kiens, B., Hoefler, G., Zechner, R., \& Zimmermann, R. (2010). Adipose triglyceride lipase plays a key role in the supply of the working muscle with fatty acids. Journal of Lipid Research, 51(3), 490-499. doi:10.1194/jr.M001073

Schweiger, M., Romauch, M., Schreiber, R., Grabner, G. F., Hütter, S., Kotzbeck, P., Benedikt, P., Eichmann, T. O., Yamada, S., Knittelfelder, O., Diwoky, C., Doler, C., Mayer, N., De Cecco, W., Breinbauer, R., Zimmermann, R., \& Zechner, R. (2017). Pharmacological inhibition of adipose triglyceride lipase corrects high-fat diet-induced insulin resistance and hepatosteatosis in mice. Nature Communications, 8(1), 14859. doi:10.1038/ncomms 14859

Shami, P. J., Saavedra, J. E., Wang, L. Y., Bonifant, C. L., Diwan, B. A., Singh, S. V., Gu, Y., Fox, S. D., Buzard, G. S., Citro, M. L., Waterhouse, D. J., Davies, K. M., Ji, X., \& Keefer, L. K. (2003). JS-K, a Glutathione/Glutathione S-Transferase-activated Nitric Oxide Donor of the Diazeniumdiolate Class with Potent Antineoplastic Activity. Molecular Cancer Therapeutics, 2(4), 409-417. Retrieved from https://mct.aacriournals.org/content/molcanther/2/4/409.full.pdf

Stamler, J. S., Lamas, S., \& Fang, F. C. (2001). Nitrosylation: The Prototypic Redox-Based Signaling Mechanism. Cell, 106(6), 675-683. doi:https://doi.org/10.1016/S00928674(01)00495-0

Tang, H.-N., Tang, C.-Y., Man, X.-F., Tan, S.-W., Guo, Y., Tang, J., Zhou, C.-L., \& Zhou, H.-D. (2017). Plasticity of adipose tissue in response to fasting and refeeding in male mice. Nutrition \& Metabolism, 14(1), 3. doi:10.1186/s12986-016-0159-x 
863

864

865

866

867

868

869

870

871

872

873

874

875

876

877

878

879

880

881

882

883

884

885

886

887

888

889

890

891

892

893

894

895

896

897

898

899

900

901

902

903

904

905

906

907

908

909

Tchernof, A., \& Després, J.-P. (2013). Pathophysiology of Human Visceral Obesity: An Update. Physiological Reviews, 93(1), 359-404. doi:10.1152/physrev.00033.2011

Velázquez, A. P., Tatsuta, T., Ghillebert, R., Drescher, I., \& Graef, M. (2016). Lipid dropletmediated ER homeostasis regulates autophagy and cell survival during starvation. The Journal of cell biology, 212(6), 621-631. doi:10.1083/jcb.201508102

Verboven, K., Wouters, K., Gaens, K., Hansen, D., Bijnen, M., Wetzels, S., Stehouwer, C. D., Goossens, G. H., Schalkwijk, C. G., Blaak, E. E., \& Jocken, J. W. (2018). Abdominal subcutaneous and visceral adipocyte size, lipolysis and inflammation relate to insulin resistance in male obese humans. Scientific Reports, 8(1), 4677. doi:10.1038/s41598018-22962-x

Vevea, Jason D., Garcia, Enrique J., Chan, Robin B., Zhou, B., Schultz, M., Di Paolo, G., McCaffery, J. M., \& Pon, Liza A. (2015). Role for Lipid Droplet Biogenesis and Microlipophagy in Adaptation to Lipid Imbalance in Yeast. Developmental Cell, 35(5), 584-599. doi:https://doi.org/10.1016/j.devcel.2015.11.010

Vieyres, G., Reichert, I., Carpentier, A., Vondran, F. W. R., \& Pietschmann, T. (2020). The ATGL lipase cooperates with ABHD5 to mobilize lipids for hepatitis $C$ virus assembly. PLOS Pathogens, 16(6), e1008554. doi:10.1371/journal.ppat.1008554

Wang, H., Quiroga, A. D., \& Lehner, R. (2013). Chapter 7 - Analysis of Lipid Droplets in Hepatocytes. In H. Yang \& P. Li (Eds.), Methods in Cell Biology (Vol. 116, pp. 107-127): Academic Press.

White, R. M., Sessa, A., Burke, C., Bowman, T., LeBlanc, J., Ceol, C., Bourque, C., Dovey, M., Goessling, W., Burns, C. E., \& Zon, L. I. (2008). Transparent adult zebrafish as a tool for in vivo transplantation analysis. Cell Stem Cell, 2(2), 183-189. doi:10.1016/j.stem.2007.11.002

Xue, Y., Lim, S., Bråkenhielm, E., \& Cao, Y. (2010). Adipose angiogenesis: quantitative methods to study microvessel growth, regression and remodeling in vivo. Nature Protocols, 5(5), 912-920. doi:10.1038/nprot.2010.46

Yamada, Y., Eto, M., Ito, Y., Mochizuki, S., Son, B.-K., Ogawa, S., lijima, K., Kaneki, M., Kozaki, K., Toba, K., Akishita, M., \& Ouchi, Y. (2015). Suppressive Role of PPARY-Regulated Endothelial Nitric Oxide Synthase in Adipocyte Lipolysis. PLoS One, 10(8), e0136597. doi:10.1371/journal.pone.0136597

Zebisch, K., Voigt, V., Wabitsch, M., \& Brandsch, M. (2012). Protocol for effective differentiation of 3T3-L1 cells to adipocytes. Analytical Biochemistry, 425(1), 88-90. doi:https://doi.org/10.1016/j.ab.2012.03.005

Zechner, R., Madeo, F., \& Kratky, D. (2017). Cytosolic lipolysis and lipophagy: two sides of the same coin. Nat Rev Mol Cell Biol, 18(11), 671-684. doi:10.1038/nrm.2017.76

Zhang, M., Di Martino, J. S., Bowman, R. L., Campbell, N. R., Baksh, S. C., Simon-Vermot, T., Kim, I. S., Haldeman, P., Mondal, C., Yong-Gonzales, V., Abu-Akeel, M., Merghoub, T., Jones, D. R., Zhu, X. G., Arora, A., Ariyan, C. E., Birsoy, K., Wolchok, J. D., Panageas, K. S., Hollmann, T., Bravo-Cordero, J. J., \& White, R. M. (2018). Adipocyte-Derived Lipids Mediate Melanoma Progression via FATP Proteins. Cancer Discovery, 8(8), 1006. doi:10.1158/2159-8290.CD-17-1371

Zimmermann, R., Strauss, J. G., Haemmerle, G., Schoiswohl, G., Birner-Gruenberger, R., Riederer, M., Lass, A., Neuberger, G., Eisenhaber, F., Hermetter, A., \& Zechner, R. (2004). Fat Mobilization in Adipose Tissue Is Promoted by Adipose Triglyceride Lipase. Science, 306, 1383-1386. 